\title{
VARIETIES WITH VANISHING HOLOMORPHIC EULER CHARACTERISTIC
}

\author{
JUNGKAI ALFRED CHEN, OLIVIER DEBARRE, AND ZHI JIANG
}

\begin{abstract}
We study smooth complex projective varieties $X$ of maximal Albanese dimension and of general type satisfying $\chi\left(X, \mathscr{O}_{X}\right)=0$. We prove that the Albanese variety of $X$ has at least three simple factors. Examples were constructed by Ein and Lazarsfeld, and we prove that in dimension 3, these examples are (up to abelian étale covers) the only ones. By results of Ueno, another source of examples is provided by varieties $X$ of maximal Albanese dimension and of general type satisfying $h^{0}\left(X, K_{X}\right)=1$. Examples were constructed by Chen and Hacon, and again, we prove that in dimension 3, these examples are (up to abelian étale covers) the only ones. We also formulate a conjecture on the general structure of these varieties in all dimensions.
\end{abstract}

\section{INTRODUCTION}

A smooth complex projective variety $X$ is said to have maximal Albanese dimension if its Albanese mapping $X \rightarrow \operatorname{Alb}(X)$ is generically finite (onto its image).

Green and Lazarsfeld showed in GL that such a variety satisfies $\chi\left(X, \omega_{X}\right) \geq 0$. Ein and Lazarsfeld later constructed in [EL] a smooth projective threefold $X$ of maximal Albanese dimension and of general type with $\chi\left(X, \omega_{X}\right)=0$ (see Examples 4.1 and 4.2).

We are interested here in describing the structure of varieties $X$ of maximal Albanese dimension (and of general type) with $\chi\left(X, \omega_{X}\right)=0$. This class of varieties is stable by modifications, étale covers, and products with any other variety of maximal Albanese dimension (and of general type). More generally, if $X$ is a smooth projective variety of maximal Albanese dimension with a fibration whose general fiber $F$ satisfies $\chi\left(F, \omega_{F}\right)=0$, then $\chi\left(X, \omega_{X}\right)=0([\mathrm{HP}]$, Proposition 2.5).

So we study smooth projective varieties $X$ of general type with $\chi\left(X, \omega_{X}\right)=0$ and a generically finite morphism $X \rightarrow A$ to an abelian variety. In \$3 , we prove a general structure theorem (Theorem 3.1) which implies among other things that $A$ has at least three simple factors. Examples where $A$ is the product of any three given non-zero factors can be constructed following Ein and Lazarsfeld, and we speculate that their construction should (more or less) describe all cases where $A$ has three simple factors but, although we prove several results in $\$ 4$ in this direction (Propositions 4.3, 4.5, and 4.7) and arrive at the rather rigid picture (5), we are only able to get a complete description when $X$ has dimension

2010 Mathematics Subject Classification. 14J10, 14J30, 14F17, 14E05.

Key words and phrases. Vanishing theorems, generic vanishing, cohomological loci, varieties of general type, Albanese dimension, Albanese variety, Euler characteristic, isotrivial fibrations.

O. Debarre is part of the project VSHMOD-2009 ANR-09- BLAN-0104-01. 
3: we prove that a smooth projective threefold $X$ of maximal Albanese dimension and of general type satisfies $\chi\left(X, \omega_{X}\right)=0$ if and only if it has an abelian étale cover which is an Ein-Lazarsfeld threefold (Theorem 5.1).

Another source of examples is provided by varieties $X$ of maximal Albanese dimension and $h^{0}\left(X, \omega_{X}\right)=1$ : it follows from work of Ueno $([\mathrm{U}])$ that they satisfy $\chi\left(X, \omega_{X}\right)=0$. Chen and Hacon constructed examples of general type (see Example 4.2). We gather some properties of these varieties in $\$ 6$. However, this class of examples is not stable under étale covers and does not lend itself well to our methods of study, except in dimension 3, where the precise Theorem 5.1 allows us to give a complete description of all smooth projective threefolds $X$ of maximal Albanese dimension and of general type, such that $P_{1}(X)=1$ : they are all modifications of abelian étale covers of Chen-Hacon threefolds (Theorem 6.3).

In 97 , we propose a conjecture on the possible general structure of smooth projective varieties $X$ of maximal Albanese dimension and of general type satisfying $\chi\left(X, \omega_{X}\right)=0$. It seems difficult to give a complete classification, but based on the examples that we know, we conjecture that, after taking modifications and étale covers, there should exists a non-trivial fibration $X \rightarrow Y$ which is either isotrivial, or whose general fiber $F$ satisfies $\chi\left(F, \omega_{F}\right)=0$. For the converse, one does have $\chi\left(X, \omega_{X}\right)=0$ in the second case by [HP], Proposition 2.5, but not necessarily in the first case, of course. Both cases do happen (Example 7.2).

We work over the field of complex numbers.

Acknowledgements. The first-named author is partially supported by NCTS and the National Science Council of Taiwan. This work started during the second-named author's visit to Taipei under the support of the bilateral Franco-Taiwanese Project Orchid and continued during the first-named author's visit to Institut Henri Poincare in Paris and the third-named author's stay at the Max Planck Institut for Mathematics in Bonn. The authors are grateful for the support they received on these occasions.

\section{Notation AND PRELIMINARIES}

For any smooth projective variety $X$, we set $\widehat{X}=\operatorname{Pic}^{0}(X)$. For $\xi \in \widehat{X}$, we will denote by $P_{\xi}$ an algebraically trivial line bundle on $X$ that represents $\xi$.

Following standard terminology, we will say that a morphism $f: X \rightarrow A$ to an abelian variety $A$ is minimal if the induced group morphism $\widehat{f}: \widehat{A} \rightarrow \widehat{X}$ is injective. Equivalently, $f(X)$ generates $A$ as an algebraic group and $f$ factors through no non-trivial abelian étale covers of $A$. The Albanese mapping $a_{X}$ has this property. Any $f: X \rightarrow A$ factors as $f: X \stackrel{f^{\prime}}{\rightarrow} A^{\prime} \rightarrow A$, where $A^{\prime}$ is an abelian variety and $f^{\prime}$ is minimal.

An algebraic fibration (or simply a fibration) is a surjective morphism between normal projective varieties, with connected fibers.

In the rest of this section, $X$ will be a smooth projective variety, of dimension $n$, with a generically finite morphism $f: X \rightarrow A$ to an abelian variety $A$. In particular, $X$ has maximal Albanese dimension. 
2.1. Cohomological loci. For each integer $i$, we define the cohomological loci

$$
\begin{aligned}
V_{i}\left(\omega_{X}, f\right) & =\left\{\xi \in \widehat{A} \mid H^{i}\left(X, \omega_{X} \otimes f^{*} P_{\xi}\right) \neq 0\right\} \\
& =\left\{\xi \in \widehat{A} \mid H^{n-i}\left(X, f^{*} P_{-\xi}\right) \neq 0\right\} .
\end{aligned}
$$

If $Y$ is a smooth projective variety and $\varepsilon: Y \rightarrow X$ is birational, we have $R^{j} \varepsilon_{*} \omega_{Y}=0$ for $j>0$ and $\varepsilon_{*} \omega_{Y} \simeq \omega_{X}\left([\mathrm{~K} 2]\right.$, Theorem 2.1), hence $\chi\left(X, \omega_{X}\right)=\chi\left(Y, \omega_{Y}\right)$ and $V_{i}\left(\omega_{X}, f\right)=$ $V_{i}\left(\omega_{Y}, f \circ \varepsilon\right)$ for all $i$. In particular, these loci do not change when $X$ is replaced with $Y$.

2.1.1. Since $R^{j} f_{*} \omega_{X}=0$ for $j>0$, we have for all $i$

$$
V_{i}\left(\omega_{X}, f\right)=V_{i}\left(f_{*} \omega_{X}\right):=\left\{\xi \in \widehat{A} \mid H^{i}\left(A, f_{*} \omega_{X} \otimes P_{\xi}\right) \neq 0\right\} .
$$

2.1.2. Each irreducible component of $V_{i}\left(\omega_{X}, f\right)$ is an abelian subvariety of $\widehat{A}$ of codimension $\geq i([\mathrm{EL}]$, Remark 1.6 and Theorem 1.2) translated by a torsion point ([Si]).

2.1.3. There is a chain of inclusions ([EL], Lemma 1.8)

$$
\operatorname{Ker}(\widehat{f})=V_{n}\left(\omega_{X}, f\right) \subseteq V_{n-1}\left(\omega_{X}, f\right) \subseteq \cdots \subseteq V_{0}\left(\omega_{X}, f\right) \subseteq \widehat{A}
$$

and $\operatorname{codim}\left(V_{n}\left(\omega_{X}, f\right)\right) \geq n$.

2.1.4. If $V_{0}\left(\omega_{X}, f\right)$ has a component of codimension $i$, this component is contained in (hence is an irreducible component of $) V_{i}\left(\omega_{X}, f\right)([\mathrm{EL}],(1.10))$, so that we have $i \leq n$ and $f(X)$ is fibered by $i$-dimensional abelian subvarieties of $A$ ([EL], Theorem 3$)$.

2.1.5. For $\xi \in \widehat{A}$ general, $\chi\left(X, \omega_{X}\right)=h^{0}\left(X, \omega_{X} \otimes f^{*} P_{\xi}\right) \geq 0$ (use 2.1.2) and

$$
\begin{aligned}
& \chi\left(X, \omega_{X}\right)=0 \Longleftrightarrow V_{0}\left(\omega_{X}, f\right) \neq \widehat{A} \\
& \Longleftrightarrow V_{0}\left(\omega_{X}, f\right) \text { has a component of codimension } i \\
& \text { for some } i \in\{1, \ldots, n-1\} \\
& \quad V_{i}\left(\omega_{X}, f\right) \text { has a component of codimension } i \\
& \text { for some } i \in\{1, \ldots, n-1\},
\end{aligned}
$$

where the last implication is not always an equivalence.

2.1.6. The variety $X$ is of general type if and only if $V_{0}\left(\omega_{X}, f\right)$ generates $\widehat{A}$ ([CH1], Theorem $2.3)$.

2.1.7. If $V_{0}\left(\omega_{X}, f\right)$ is finite, Ein and Lazarsfeld proved that $X$ is birational to an abelian variety ([CH1], Theorem 1.3.2). In particular, if $f$ is moreover minimal, it is a birational isomorphism. 
2.2. Composing $f$ with a generically finite morphism. If $Y$ is smooth projective and $h: Y \rightarrow X$ is surjective and generically finite, the trace map $h_{*} \omega_{Y} \rightarrow \omega_{X}$ splits the natural inclusion $\omega_{X} \rightarrow h_{*} \omega_{Y}$, hence $H^{i}\left(X, \omega_{X} \otimes f^{*} P_{\xi}\right)$ injects into $H^{i}\left(X, h_{*} \omega_{Y} \otimes f^{*} P_{\xi}\right)$ for all $\xi \in \widehat{A}$. Thus, using also 2.1.1, we obtain

$$
V_{i}\left(\omega_{X}, f\right) \subseteq V_{i}\left(h_{*} \omega_{Y}, f\right)=V_{i}\left(\omega_{Y}, f \circ h\right) .
$$

Morover,

$$
\chi\left(Y, \omega_{Y}\right) \geq \chi\left(X, \omega_{X}\right) .
$$

When $h$ is étale, we have

$$
\chi\left(Y, \omega_{Y}\right)=\operatorname{deg}(h) \chi\left(X, \omega_{X}\right) .
$$

Finally, when $h$ is obtained from an isogeny $\eta: B \rightarrow A$ as in the cartesian diagram

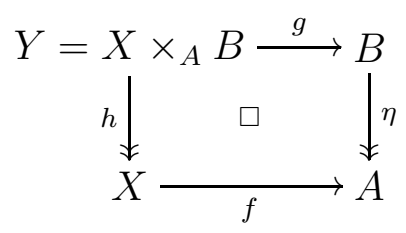

we have $V_{i}\left(\omega_{Y}, g\right)=\widehat{\eta}\left(V_{i}\left(\omega_{X}, f\right)\right)$. Combining this with 2.1.2, we see that after making a suitable étale base change, we can always make all the components of $V_{i}\left(\omega_{X}, f\right)$ pass through 0 .

\section{Components of $V_{i}$ OF Codimension $i$}

Let $X$ be a smooth projective variety with a generically finite morphism $f: X \rightarrow A$ to an abelian variety. If $\chi\left(X, \omega_{X}\right)=0$, it follows from 2.1.5 that $V_{i}\left(\omega_{X}, f\right)$ has a component of codimension $i$ for some $i \in\{1, \ldots, n-1\}$. We prove a structure theorem under this weaker assumption.

Theorem 3.1. Let $X$ be a smooth projective variety of dimension $n$, let $A$ be an abelian variety, and let $f: X \rightarrow A$ be a minimal generically finite morphism. Assume that for some $i \in\{0, \ldots, n\}$, the locus $V_{i}\left(\omega_{X}, f\right)$ has a component $V$ of codimension $i$ in $\widehat{A}$. Let $B$ be the abelian variety $\widehat{V}$, let $K:=\operatorname{Ker}(A \rightarrow B)^{0}$, and assume $f(X)+K=f(X)$. For a suitable modification $X^{\prime}$ of an abelian étale cover of $X$, the Stein factorization of the morphism $X^{\prime} \rightarrow A \rightarrow B$ induces a surjective morphism $X^{\prime} \rightarrow Y$ where $Y$ is smooth of dimension $n-i$, of general type, with $\chi\left(Y, \omega_{Y}\right)>0$.

Remark 3.2. The condition $f(X)+K=f(X)$ holds:

- when $f$ is surjective;

- when $V$ is also a component of $V_{0}\left(\omega_{X}, f\right)$ ([EL], proof of Theorem 3$)$; this applies in particular when $\chi\left(X, \omega_{X}\right)=0$ (2.1.5).

Proof of Theorem 3.1. By (2.1.2) and $\$ 2.2$, we may assume, after isogeny, that $A=B \times K$ and $V=\widehat{B}$. Let $p: A \rightarrow B$ be the projection. Considering the Stein factorization of 
$\pi=p \circ f: X \rightarrow B$, and replacing $X$ by a suitable modification, we may assume that $\pi$ factors as

$$
\pi: X \stackrel{g}{\rightarrow} Y \stackrel{h}{\rightarrow} B
$$

where $Y$ is smooth, $h$ is generically finite, and $g$ is surjective with connected fibers. Since $f(X)+K=f(X)$, the image of $\pi$ has dimension $\operatorname{dim}(X)-\operatorname{dim}(K)$, hence general fibers of $g$ have dimension $\operatorname{dim}(K)=i$.

We then have $R^{i} g_{*} \omega_{X} \simeq \omega_{Y}$ ([K2], Proposition 7.6). Moreover, the sheaves $R^{k} g_{*} \omega_{X}$ on $Y$ satisfy the generic vanishing theorem ([HP], Theorem 2.2), hence

$$
V_{j}\left(R^{k} g_{*} \omega_{X}, h\right) \neq \widehat{B} \quad \text { for all } j>0 \text { and all } k \text {. }
$$

For all

$$
\xi \in \widehat{B}-\bigcup_{j>0, k} V_{j}\left(R^{k} g_{*} \omega_{X}, h\right)
$$

we have

$$
H^{j}\left(Y, R^{k} g_{*} \omega_{X} \otimes h^{*} P_{\xi}\right)=0 \quad \text { for all } j>0 \text { and all } k .
$$

Hence, by the Leray spectral sequence, we obtain

$$
h^{i}\left(X, \omega_{X} \otimes f^{*} P_{\xi}\right)=h^{i}\left(X, \omega_{X} \otimes \pi^{*} P_{\xi}\right)=h^{0}\left(Y, R^{i} g_{*} \omega_{X} \otimes h^{*} P_{\xi}\right)=h^{0}\left(Y, \omega_{Y} \otimes h^{*} P_{\xi}\right)
$$

and these numbers are non-zero because $\widehat{B}=V \subseteq V_{i}\left(\omega_{X}, f\right)$. In particular, $V_{0}\left(\omega_{Y}, h\right)=\widehat{B}$. By 2.1.6 and 2.1.5, $Y$ is of general type and $\chi\left(Y, \omega_{Y}\right)>0$. This completes the proof.

We prove a partial converse to Theorem 3.1: assume that there is a generically finite morphism $f: X \rightarrow A$ and a quotient abelian variety $A \rightarrow B$ such that $f(X)+K=f(X)$, where $K:=\operatorname{Ker}(A \rightarrow B)^{0}$, and denote by $X \rightarrow Y \rightarrow B$ a modification of the Stein factorization of $X \rightarrow A \rightarrow B$, where $Y$ is smooth of dimension $n-i$ (we set $i:=\operatorname{dim}(K)$ ).

Proposition 3.3. In this situation, if $Y$ is not birational to an abelian variety, $V_{j}\left(\omega_{X}, f\right)$ has a component of codimension $j$ for some $j \in\{i, \ldots, n-1\}$.

Proof. Replacing $X$ with a modification of an étale cover (which is allowed by $\$ 2.1$ and $\$ 2.2$ ), we may assume that we have a factorization

$$
f: X \stackrel{(g, k)}{\longrightarrow} Y \times K \stackrel{h \times \operatorname{Id}_{K}}{\longrightarrow} B \times K,
$$

where $(g, k)$ is surjective and $h: Y \rightarrow B$ is generically finite of degree $>1$. We obtain, as in the proof of Theorem 3.1, for $\xi$ general in $\widehat{B}$,

$$
h^{i}\left(X, \omega_{X} \otimes f^{*} P_{\xi}\right)=h^{0}\left(Y, R^{i} g_{*} \omega_{X} \otimes h^{*} P_{\xi}\right)=h^{0}\left(Y, \omega_{Y} \otimes h^{*} P_{\xi}\right) .
$$

If $\chi\left(Y, \omega_{Y}\right)>0$, we have $V_{0}\left(\omega_{Y}, h\right)=\widehat{B}$, the number on the right-hand-side of (2) is non-zero for all $\xi$, hence $V_{i}\left(\omega_{X}, f\right)$ contains the $i$-codimensional abelian subvariety $\widehat{B}$ of $\widehat{A}$.

If $\chi\left(Y, \omega_{Y}\right)=0$, since $Y$ is not birational to an abelian variety, $V_{0}\left(\omega_{Y}, h\right)$ has (by 2.1.7 and 2.1.5) a component of codimension $l \in\{1, \ldots, n-i-1\}$ in $\widehat{B}$. Thus, by Remark 3.2 , we can apply Theorem 3.1 to $h: Y \rightarrow B$ : after taking an étale cover and a modification, $h$ factors through a morphism $Y \rightarrow Z \times C$, where $C$ is an abelian variety of dimension $l$, 
$\chi\left(Z, \omega_{Z}\right)>0$, and $\operatorname{dim}(Z)=n-i-l$. We are therefore reduced to the first case and we conclude again that $V_{i+l}\left(\omega_{X}, f\right)$ contains an $(i+l)$-codimensional component.

Remark 3.4. Under the hypotheses of Theorem 3.1 and the assumption $A=B \times K$ made in its proof, we obtain a surjective morphism $k: X \stackrel{f}{\rightarrow} B \times K \stackrel{p_{2}}{\rightarrow} K$ and, from its Stein factorization, morphisms

$$
k: X \stackrel{l}{\rightarrow} Z \stackrel{m}{\rightarrow} K,
$$

where $Z$ is smooth of dimension $i, m$ is generically finite, and $l$ has connected (generically $(n-i)$-dimensional) fibers. We have again $R^{n-i} l_{*} \omega_{X} \simeq \omega_{Z}$ and, for $\xi$ general in $\widehat{K}$,

$$
h^{n-i}\left(X, \omega_{X} \otimes f^{*} P_{\xi}\right)=h^{0}\left(Z, \omega_{Z} \otimes m^{*} P_{\xi}\right) .
$$

Then,

a) either $V_{0}\left(\omega_{Z}, m\right) \subsetneq \widehat{K}$ and $\chi\left(Z, \omega_{Z}\right)=0$;

b) or $V_{0}\left(\omega_{Z}, m\right)=\widehat{K}$ and $\chi\left(Z, \omega_{Z}\right)>0$, in which case $\widehat{K}$ is contained in (hence is a component of $) V_{n-i}\left(\omega_{X}, f\right)$. There is a surjective generically finite map $X \rightarrow Y \times Z$, hence $\chi\left(X, \omega_{X}\right) \geq \chi\left(Y, \omega_{Y}\right) \chi\left(Z, \omega_{Z}\right)>0$ (this also follows from Corollary [3.5. a) below).

Finally, if $F$ is a general fiber of $l: X \rightarrow Z$, there is a surjective generically finite map $F \rightarrow Y$, hence $\chi\left(F, \omega_{F}\right)>0($ see (10) $)$.

We now deduce some consequences of Theorem 3.1 on the possible components of $V_{0}\left(\omega_{X}, f\right)$ and the number of simple factors of the abelian variety $A$.

Corollary 3.5. Let $X$ be a smooth projective variety with $\chi\left(X, \omega_{X}\right)=0$ and a generically finite morphism $f: X \rightarrow A$ to an abelian variety.

a) The locus $V_{0}\left(\omega_{X}, f\right)$ does not have complementary components 1

b) If $X$ is in addition of general type, A has at least three simple factors.

Proof. If $V_{0}\left(\omega_{X}, f\right)$ has complementary components $V_{1}, \ldots, V_{r}$, with duals $B_{1}, \ldots, B_{r}$, the image $f(X)$ is stable by translation by $\prod_{j \neq i} B_{j}$ for each $i$ (Remark 3.2), hence $f$ is surjective if $r \geq 2$. We obtain from Theorem 3.1, after passing to an étale cover and a modification of $X$, a generically finite surjective map $X \rightarrow Y_{1} \times \cdots \times Y_{r}$, with $\chi\left(Y_{i}, \omega_{Y_{i}}\right)>0$ for all $i$. Since $\chi\left(X, \omega_{X}\right) \geq \prod_{i} \chi\left(Y_{i}, \omega_{Y_{i}}\right)$ (by (1) ), this is absurd. This proves a). Item $\mathrm{b}$ ) then follows from 2.1.6.

Proposition 3.6. Let $X$ be a smooth projective variety of general type, with $\chi\left(X, \omega_{X}\right)=0$ and a generically finite morphism $f: X \rightarrow A$ to an abelian variety. Then $V_{0}\left(\omega_{X}, f\right)$ has no 1-dimensional components.

Proof. Assume $V_{0}\left(\omega_{X}, f\right)$ has a one-dimensional component and write it as $\tau_{1}+\widehat{B}_{1}$ for some torsion point $\tau_{1} \in \widehat{A}$ and some quotient elliptic curve $A \rightarrow B_{1}$. By [J], Proposition 1.7, and since $V_{0}\left(\omega_{X}, f\right)$ generates $\widehat{A}(\underline{2.1 .6}), \widehat{B}_{1}$ cannot be maximal for inclusion: more precisely,

\footnotetext{
$\widehat{A}$.

${ }^{1}$ By that, we mean components such that the sum morphism induces an isogeny from their product onto
} 
there must exist two maximal components (in the sense of [J], Definition 1.6) $\tau_{2}+\widehat{B}_{2}$ and $\tau_{3}+\widehat{B}_{3}$ of $V_{0}\left(\omega_{X}, f\right)$ with $\widehat{B}_{1} \subsetneq \widehat{B}_{j} \subsetneq \widehat{A}$ for each $j \in\{2,3\}$ and $\widehat{B}_{2} \neq \widehat{B}_{3}$. There are corresponding factorizations $A \rightarrow B_{j} \rightarrow B_{1}$.

As in the proof of Theorem 3.1, after passing to an étale cover and a modification of $X$, we may assume $\tau_{1}=\tau_{2}=\tau_{3}=0$ and that we have

- Stein factorizations

$$
X \stackrel{g_{i}}{\longrightarrow} Y_{i} \stackrel{h_{i}}{\longrightarrow} B_{i}
$$

where $h_{1}, h_{2}$, and $h_{3}$ are generically finite, $Y_{1}, Y_{2}$, and $Y_{3}$ are smooth, $Y_{1}$ is a curve of genus $\geq 2$, and $\chi\left(Y_{2}, \omega_{Y_{2}}\right)$ and $\chi\left(Y_{3}, \omega_{Y_{3}}\right)$ are both positive (Theorem [3.1),

- a commutative diagram

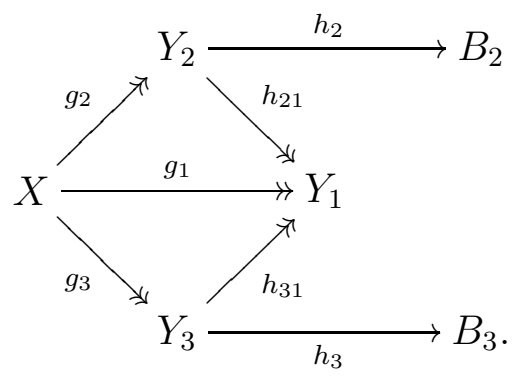

We may further assume that the induced morphism $X \rightarrow Y_{2} \times_{Y_{1}} Y_{3}$ factors as:

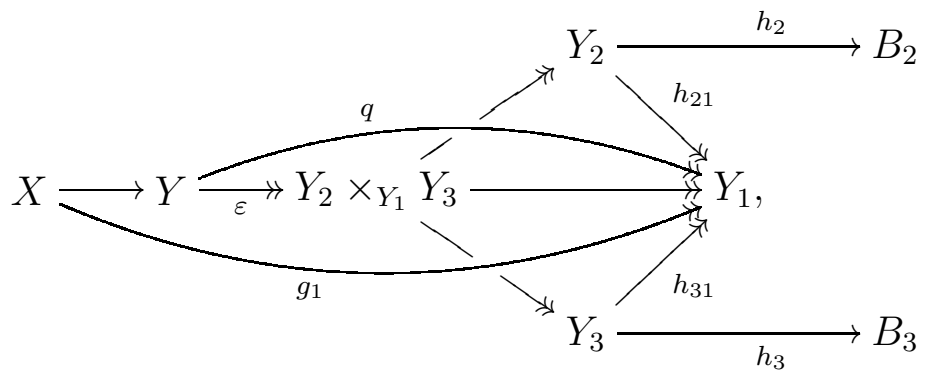

where $\varepsilon$ is a resolution of singularities.

Now take $\xi_{2} \in \widehat{B}_{2}$ and $\xi_{3} \in \widehat{B}_{3}$. By [M], Lemma 4.10.(ii) [2 there is an inclusion

$$
q_{*}\left(\omega_{Y / Y_{1}} \otimes \varepsilon^{*}\left(h_{2}^{*} P_{\xi_{2}} \otimes h_{3}^{*} P_{\xi_{3}}\right)\right) \subseteq h_{21 *}\left(\omega_{Y_{2} / Y_{1}} \otimes h_{2}^{*} P_{\xi_{2}}\right) \otimes h_{31 *}\left(\omega_{Y_{3} / Y_{1}} \otimes h_{3}^{*} P_{\xi_{3}}\right),
$$

of locally free sheaves of the same rank on the curve $Y_{1}$. Moreover, we saw during the proof of Theorem 3.1 that for $j \in\{2,3\}$, we have

$$
0 \neq h^{0}\left(Y_{j}, \omega_{Y_{j}} \otimes h_{j}^{*} P_{\xi_{j}}\right)=h^{0}\left(Y_{1}, h_{j 1 *}\left(\omega_{Y_{j}} \otimes h_{j}^{*} P_{\xi_{j}}\right)\right) .
$$

It follows that the sheaf $h_{j 1 *}\left(\omega_{Y_{j}} \otimes h_{j}^{*} P_{\xi_{j}}\right)$ is non-zero, hence so is the sheaf $h_{j 1 *}\left(\omega_{Y_{j} / Y_{1}} \otimes h_{j}^{*} P_{\xi_{j}}\right)$. All in all, we have obtained that the locally free sheaf $q_{*}\left(\omega_{Y / Y_{1}} \otimes \varepsilon^{*}\left(h_{2}^{*} P_{\xi_{2}} \otimes h_{3}^{*} P_{\xi_{3}}\right)\right)$ is non-zero.

Assume now that $\xi_{2}$ and $\xi_{3}$ are torsion. By [V], Corollary 3.6.3 this vector bundle is nef, hence has non-negative degree. Since $Y_{1}$ is a curve of genus $\geq 2$, the Riemann-Roch

\footnotetext{
${ }^{2}$ This is stated in $[\mathrm{M}]$ for $\xi_{2}=\xi_{3}=0$, but the same proof works in general.

${ }^{3}$ This is stated there for $\xi_{2}=\xi_{3}=0$, but the general case follows by the étale covering trick.
} 
theorem then implies

$$
0 \neq h^{0}\left(Y_{1}, q_{*}\left(\omega_{Y} \otimes \varepsilon^{*}\left(h_{2}^{*} P_{\xi_{2}} \otimes h_{3}^{*} P_{\xi_{3}}\right)\right)\right)=h^{0}\left(Y, \omega_{Y} \otimes \varepsilon^{*}\left(h_{2}^{*} P_{\xi_{2}} \otimes h_{3}^{*} P_{\xi_{3}}\right)\right) .
$$

Finally, note that both $X$ and $Y$ have maximal Albanese dimensions. This implies that $\omega_{X / Y}$ is effective, hence $h^{0}\left(X, \omega_{X} \otimes f^{*}\left(P_{\xi_{2}} \otimes P_{\xi_{3}}\right)\right)$ is also non-zero. It follows that $\xi_{2}+\xi_{3}$ is in $V_{0}\left(\omega_{X}, f\right)$, which therefore contains $\widehat{B}_{2}+\widehat{B}_{3}$. This contradicts the fact that $\widehat{B}_{2}$ is maximal.

\section{Case when $A$ has three simple faCtors}

Ein and Lazarsfeld constructed an example of a smooth projective threefold $X$ of maximal Albanese dimension and of general type with $\chi\left(X, \omega_{X}\right)=0$, whose Albanese variety is the product of three elliptic curves. After presenting their construction (and a variant due to Chen and Hacon), we prove some general results when $\operatorname{Alb}(X)$ has three simple factors. In the next section, we will show that the Ein-Lazarsfeld example is essentially the only one in dimension 3 (Theorem 5.1).

Example 4.1 ([EL], Example 1.13). Let $E_{1}, E_{2}$, and $E_{3}$ be elliptic curves and let $\rho_{j}: C_{j} \rightarrow$ $E_{j}$ be double coverings, where $C_{j}$ is a smooth curve of genus $\geq 2$ and $\rho_{j *} \omega_{C_{j}} \simeq \mathscr{O}_{E_{j}} \oplus \delta_{j}$. Denote by $\iota_{j}$ the corresponding involution of $C_{j}$. Let $A=E_{1} \times E_{2} \times E_{3}$, and consider the quotient $Z$ of $C_{1} \times C_{2} \times C_{3}$ by the involution $\iota_{1} \times \iota_{2} \times \iota_{3}$ and the tower of Galois covers:

$$
C_{1} \times C_{2} \times C_{3} \stackrel{g}{\longrightarrow} Z \stackrel{f}{\longrightarrow} A
$$

of degrees 2 and 4 respectively. Observe that $Z$ has rational singularities and is minimal of general type. Let $\varepsilon: X \rightarrow Z$ be any desingularization. The Albanese map of $X$ is $a_{X}=f \circ \varepsilon$ and

$$
a_{X *} \omega_{X} \simeq \mathscr{O}_{A} \oplus\left(L_{1} \otimes L_{2}\right) \oplus\left(L_{3} \otimes L_{1}\right) \oplus\left(L_{2} \otimes L_{3}\right),
$$

where $L_{j}$ is the inverse image of $\delta_{j}$ by the projection $A \rightarrow E_{j}$, hence

$$
V_{0}\left(\omega_{X}, a_{X}\right)=V_{1}\left(\omega_{X}, a_{X}\right)=\left(\widehat{E}_{1} \times \widehat{E}_{2} \times\{0\}\right) \cup\left(\widehat{E}_{1} \times\{0\} \times \widehat{E}_{3}\right) \cup\left(\{0\} \times \widehat{E}_{2} \times \widehat{E}_{3}\right),
$$

whereas $V_{2}\left(\omega_{X}, a_{X}\right)=V_{3}\left(\omega_{X}, a_{X}\right)=\{0\}$.

This provides three-dimensional examples. Obviously, the same construction works starting from double coverings $\rho_{j}: X_{j} \rightarrow A_{j}$ of abelian varieties with smooth ample branch loci and provides examples in all dimensions $\geq 3$. One can also extend it to any odd number $2 r+1$ of factors and get examples where the Albanese mapping is birationally a $(\mathbf{Z} / 2 \mathbf{Z})^{2 r}$ covering.

Example 4.2 ([CH2], §4, Example). A variant of the construction above was given by Chen and Hacon. Keeping the same notation, choose points $\xi_{j} \in \widehat{E}_{j}$ of order 2 and consider the induced double étale covers $C_{j}^{\prime} \rightarrow C_{j}$, with associated involution $\sigma_{j}$, and $E_{j}^{\prime} \rightarrow E_{j}$. The involution $\iota_{j}$ on $C_{j}$ pulls back to an involution $\iota_{j}^{\prime}$ on $C_{j}^{\prime}$ (with quotient $E_{j}^{\prime}$ ). Let $Z^{\prime}$ be the quotient of $C_{1}^{\prime} \times C_{2}^{\prime} \times C_{3}^{\prime}$ by the group of automorphisms generated by $\mathrm{id}_{1} \times \sigma_{2} \times \iota_{3}^{\prime}$, $\iota_{1}^{\prime} \times \mathrm{id}_{2} \times \sigma_{3}, \sigma_{1} \times \iota_{2}^{\prime} \times \mathrm{id}_{3}$, and $\sigma_{1} \times \sigma_{2} \times \sigma_{3}$, and let $\varepsilon^{\prime}: X^{\prime} \rightarrow Z^{\prime}$ be a desingularization. There is a morphism $f^{\prime}: X^{\prime} \rightarrow A$ of degree 4 , the Albanese map of $X^{\prime}$ is $a_{X^{\prime}}=f^{\prime} \circ \varepsilon^{\prime}$, and

$$
a_{X^{\prime} *} \omega_{\tilde{X}^{\prime}} \simeq \mathscr{O}_{A} \oplus\left(L_{1} \otimes L_{2}^{\xi} \otimes P_{\xi_{3}}\right) \oplus\left(L_{1}^{\xi} \otimes P_{\xi_{2}} \otimes L_{3}\right) \oplus\left(P_{\xi_{1}} \otimes L_{2} \otimes L_{3}^{\xi}\right),
$$


where $L_{j}^{\xi}=L_{j} \otimes P_{\xi_{j}}$. In particular, $P_{1}\left(X^{\prime}\right)=1$, and

$V_{0}\left(\omega_{X^{\prime}}, a_{X^{\prime}}\right)=V_{1}\left(\omega_{X^{\prime}}, a_{X^{\prime}}\right)=\{0\} \cup\left(\widehat{E}_{1} \times \widehat{E}_{2} \times\left\{\xi_{3}\right\}\right) \cup\left(\widehat{E}_{1} \times\left\{\xi_{2}\right\} \times \widehat{E}_{3}\right) \cup\left(\left\{\xi_{1}\right\} \times \widehat{E}_{2} \times \widehat{E}_{3}\right)$.

Of course, the étale cover $E_{1}^{\prime} \times E_{2}^{\prime} \times E_{3}^{\prime} \rightarrow E_{1} \times E_{2} \times E_{3}$ pulls back to an étale cover $X^{\prime \prime} \rightarrow X^{\prime}$, where $X^{\prime \prime}$ is an Ein-Lazarsfeld threefold.

Again, this construction still works starting from double coverings of abelian varieties with smooth ample branch loci, providing examples in all dimensions $\geq 3$, and for any odd number $2 r+1$ of factors, providing examples where the Albanese mapping is birationally a $(\mathbf{Z} / 2 \mathbf{Z})^{2 r}$-covering.

Proposition 4.3. Let $X$ be a smooth projective variety of general type with $\chi\left(X, \omega_{X}\right)=0$ and a generically finite morphism $f: X \rightarrow A$ to an abelian variety $A$ with exactly three simple factors $A_{1}, A_{2}, A_{3}$.

a) The map $f$ is surjective.

b) After passing to an abelian étale cover, we may assume $A=A_{1} \times A_{2} \times A_{3}$ and that $\widehat{A}_{1} \times \widehat{A}_{2} \times\{0\}, \widehat{A}_{1} \times\{0\} \times \widehat{A}_{3}$, and $\{0\} \times \widehat{A}_{2} \times \widehat{A}_{3}$ are irreducible components of $V_{0}\left(\omega_{X}, f\right)$.

Proof. We begin with the proof of item b). As in the proof of Theorem [3.1, we may assume, after passing to an abelian étale cover, that $A$ is $A_{1} \times A_{2} \times A_{3}$ and, by Corollary 3.5. a) and 2.1.6, that $\widehat{A}_{1} \times \widehat{A}_{2} \times\{0\}$ and $\widehat{A}_{1} \times\{0\} \times \widehat{A}_{3}$ are irreducible components of $V_{0}\left(\omega_{X}, f\right)$.

Assume that the projection $V_{0}\left(\omega_{X}, f\right) \rightarrow \widehat{A}_{2} \times \widehat{A}_{3}$ is not surjective. For $\xi_{2}$ and $\xi_{3}$ general torsion points in $\widehat{A}_{2}$ and $\widehat{A}_{3}$ respectively, we then have

$$
\left(\xi_{2}+\xi_{3}+\widehat{A}_{1}\right) \cap V_{0}\left(\omega_{X}, f\right)=\varnothing .
$$

Consider the morphism $f_{1}=p_{1} \circ f: X \rightarrow A_{1}$ and the sheaf $\mathscr{E}=f_{1 *}\left(\omega_{X} \otimes f_{2}^{*} P_{\xi_{2}} \otimes f_{3}^{*} P_{\xi_{3}}\right)$ on $A_{1}$. By [HP], Theorem 2.2, the cohomological loci of $\mathscr{E}$ satisfy the chain of inclusions 2.1.3. On the other hand, for all $\xi \in \widehat{A}_{1}$, we have $H^{0}\left(A_{1}, \mathscr{E} \otimes P_{\xi}\right)=0$, hence $V_{0}(\mathscr{E})=\varnothing$. It follows that for all $\xi \in \widehat{A}_{1}$ and all $i \geq 0$, we have $H^{i}\left(A_{1}, \mathscr{E} \otimes P_{\xi}\right)=0$. This implies $\mathscr{E}=0$ by Fourier-Mukai duality $([\mathrm{Mu}])$. But the rank of $\mathscr{E}$ is at least $\chi\left(F_{1}, \omega_{F_{1}}\right)$, where $F_{1}$ is a component of a general fiber of $f_{1}\left([\mathrm{HP}]\right.$, Corollary 2.3) and this is impossible: $F_{1}$ is of general type and generically finite over $A_{2} \times A_{3}$, hence $\chi\left(F_{1}, \omega_{F_{1}}\right)>0$ (Corollary 3.5.b)).

The projection $V_{0}\left(\omega_{X}, f\right) \rightarrow \widehat{A}_{2} \times \widehat{A}_{3}$ is therefore surjective. Since $A_{1}$ is simple, this implies that, after passing to a (split) étale cover of $A$, there are morphisms $u_{2}: \widehat{A}_{2} \rightarrow \widehat{A}_{1}$ and $u_{3}: \widehat{A}_{3} \rightarrow \widehat{A}_{1}$ such that

$$
\left\{u_{2}\left(\xi_{2}\right)+u_{3}\left(\xi_{3}\right)+\xi_{2}+\xi_{3} \mid \xi_{2} \in \widehat{A}_{2}, \xi_{3} \in \widehat{A}_{3}\right\}
$$

is a component of $V_{0}\left(\omega_{X}, f\right)$. Composing this cover with the automorphism $\left(a_{1}, a_{2}, a_{3}\right) \mapsto$ $\left(a_{1}, a_{2}-\widehat{u}_{2}\left(a_{1}\right), a_{3}-\widehat{u}_{3}\left(a_{1}\right)\right)$ of $A$, we obtain b).

Item a) then follows from the fact that $f(X)$ is stable by translation by each $A_{i}$ (Remark 3.2) hence is equal to $A$. 
Remark 4.4. As shown by considering the product with a curve of genus $\geq 2$ of any variety $X$ of general type and maximal Albanese dimension with $\chi\left(X, \omega_{X}\right)=0$, the conclusion of Proposition [4.3. a) does not hold in general as soon as $A$ has at least four simple factors.

Proposition 4.5. Let $X$ be a smooth projective variety of general type of dimension $n$ with $\chi\left(X, \omega_{X}\right)=0$ and a generically finite morphism $X \rightarrow A$ to an abelian variety $A$ with exactly three simple factors. We have:

a) $q(X)=n$;

b) the general fiber $F$ of any non-constant fibration $X \rightarrow Y$ satisfies $\chi\left(F, \omega_{F}\right)>0$;

c) any morphism from $X$ to a curve of genus $\geq 2$ is constant;

d) $V_{n-1}\left(\omega_{X}, a_{X}\right)=\{0\}$.

Proof. Let us prove b) first. The fiber $F$ is of general type and is generically finite but not surjective over $A$, hence $\chi\left(F, \omega_{F}\right)>0$ by Proposition 4.3. a). The other items then follow from the lemma below.

Lemma 4.6. Let $X$ be a smooth projective variety of dimension n, of maximal Albanese dimension, of general type, with $\chi\left(X, \omega_{X}\right)=0$. Assume that the general fiber $F$ of any non-constant fibration $X \rightarrow Y$ satisfies $\chi\left(F, \omega_{F}\right)>0$. Then,

a) the Albanese mapping $a_{X}$ is surjective $(q(X)=n)$;

b) any morphism from $X$ to a curve of genus $\geq 2$ is constant;

c) $V_{n-1}\left(\omega_{X}, a_{X}\right)=\{0\}$.

Proof. Item a) follows from [CH2], Theorem 4.2, and item b) from [HP], Theorem 2.4. Let us prove c).

If $V_{n-1}\left(\omega_{X}, a_{X}\right)-\{0\}$ is non-empty, it contains a torsion point by 2.1.2, which defines a connected étale cover $\pi: \widetilde{X} \rightarrow X$ such that $q(\widetilde{X})>q(X)=n$. By [CH2], Theorem 4.2, again, there exists a non-constant fibration $\widetilde{X} \rightarrow Y$ with general fiber $F$ of maximal Albanese dimension, of general type, with $\chi\left(F, \omega_{F}\right)=0$, such that $a_{\tilde{X}}(F)$ is a translate of a fixed abelian subvariety $\widetilde{K}$ of $\operatorname{Alb}(\widetilde{X})$ and $\operatorname{dim}(\widetilde{K})=\operatorname{dim}(F)<\operatorname{dim}(X)$. We consider the image $K$ of $\widetilde{K}$ by the induced map $\operatorname{Alb}(\pi): \operatorname{Alb}(\widetilde{X}) \rightarrow \operatorname{Alb}(X)$ and the commutative diagram:

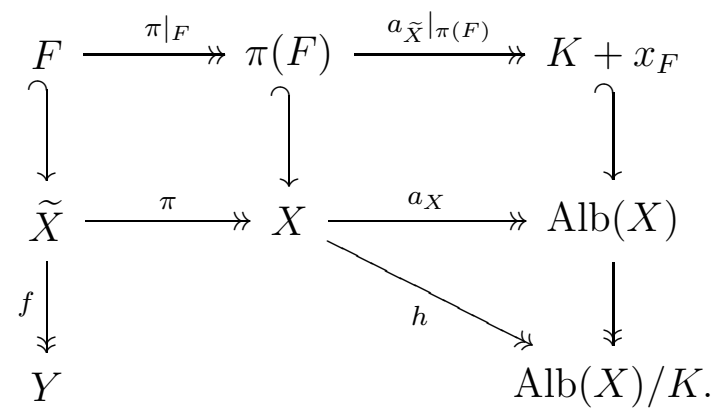

The map $\left.a_{\tilde{X}}\right|_{\pi(F)}$ is generically finite, hence $\operatorname{dim}(K)=\operatorname{dim} \pi(F)=\operatorname{dim}(F)$, and

$$
\operatorname{dim}(\operatorname{Alb}(X) / K)=n-\operatorname{dim}(K)=n-\operatorname{dim}(F) .
$$


It follows that $\pi(F)$ is a general fiber of the Stein factorization of $h$. Since $\chi\left(\pi(F), \omega_{\pi(F)}\right)=0$, this contradicts our hypothesis on $X$.

Assume now that we are in the situation of Proposition 4.3. b) and consider, as in Remark 3.4, the maps $f_{i}:=p_{i} \circ f: X \rightarrow A_{i}$. Since $\chi\left(X, \omega_{X}\right)=0$, we are in case a) of that remark, hence, with the notation therefrom, $V_{0}\left(\omega_{Z}, m\right)$ must be finite, because $A_{i}$ is simple. If $f$ is minimal, so is $f_{i}$ and it follows from 2.1.7 that $Z$ is birational to $A_{i}$, hence $f_{i}$ is a fibration. A general fiber $F_{i}$ satisfies $\chi\left(F_{i}, \omega_{F_{i}}\right)>0$ by Proposition 4.5.b).

Proposition 4.7. Let $X$ be a smooth projective variety of general type with $\chi\left(X, \omega_{X}\right)=0$ and a minimal generically finite morphism $f: X \rightarrow A$ to an abelian variety $A$ product of three simple factors $A_{1}, A_{2}$, and $A_{3}$.

Let $\{i, j, k\}=\{1,2,3\}$. For $\xi_{j}$ and $\xi_{k}$ general torsion points in $\widehat{A}_{j}$ and $\widehat{A}_{k}$ respectively, the sheaf $f_{i *}\left(\omega_{X} \otimes f_{j}^{*} P_{\xi_{j}} \otimes f_{k}^{*} P_{\xi_{k}}\right)$ on $A_{i}$ is locally free, homogeneous, of positive rank $\chi\left(F_{i}, \omega_{F_{i}}\right)$.

Proof. We follow the proof of [CH2], Corollary 2.3. As in the proof of Proposition 4.3, since $\xi_{j}$ and $\xi_{k}$ are torsion, the cohomological loci of $\mathscr{E}:=f_{i *}\left(\omega_{X} \otimes f_{j}^{*} P_{\xi_{j}} \otimes f_{k}^{*} P_{\xi_{k}}\right)$ satisfy the chain of inclusions 2.1.3. On the other hand, since $\xi_{j}$ and $\xi_{k}$ are general and $A_{i}$ is simple, the intersection

$$
\left(\xi_{j}+\xi_{k}+\widehat{A}_{i}\right) \cap V_{0}\left(\omega_{X}, f\right)
$$

is finite by 2.1.2, hence so is $V_{0}(\mathscr{E})$. It follows that all $V_{l}(\mathscr{E})$ are finite, hence $\mathscr{E}$ is locally free and homogeneous $\left([\mathrm{Mu}]\right.$, Example 3.2). Its rank is $h^{0}\left(F_{i}, \omega_{F_{i}} \otimes f_{j}^{*} P_{\xi_{j}} \otimes f_{k}^{*} P_{\xi_{k}}\right)=\chi\left(F_{i}, \omega_{F_{i}}\right)$.

Remark 4.8. Recall that a homogeneous vector bundle is a direct sum of twists of unipotent vector bundles (successive extensions of trivial line bundles) by algebraically trivial line bundles which, in our case, are torsion by Simpson's theorem (or rather its extension [HP], Theorem 2.2.b)).

When $A_{i}$ is an elliptic curve, the sheaf of Proposition 4.7 is actually a direct sum of torsion line bundles (this is explained at the bottom of page 362 of [K3] when $\xi_{j}=\xi_{k}=0$ and holds in general by the étale covering trick).

Finally, from the proof of Theorem 3.1, we have (after replacing $X$ with a suitable modification), for each $\{i, j, k\}=\{1,2,3\}$, Stein factorizations

$$
p_{j k} \circ f: X \stackrel{g_{i}}{\longrightarrow} S_{i} \stackrel{\left(h_{i j}, h_{i k}\right)}{\longrightarrow} A_{j} \times A_{k},
$$

where $S_{i}$ is smooth of general type with $\chi\left(S_{i}, \omega_{S_{i}}\right)>0$ (this follows also from Corollary 3.5.b)). Since $f_{j}$ has connected fibers, so does $h_{i j}: S_{i} \rightarrow A_{j}$. 
All in all, we have for each $\{i, j, k\}=\{1,2,3\}$ a commutative diagram:

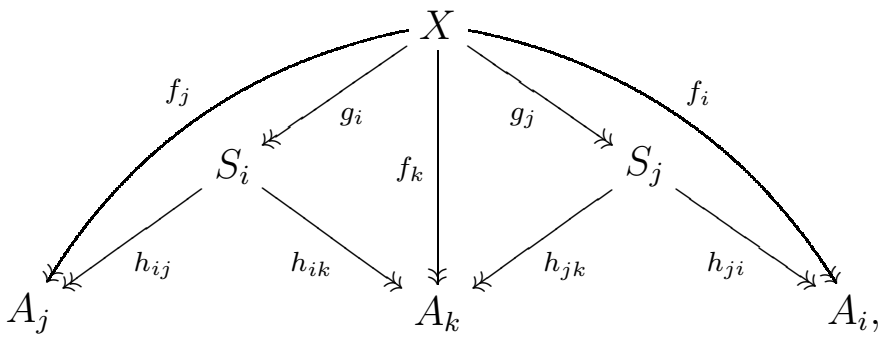

where all the morphisms are fibrations.

Question 4.9. Are the $h_{i j}$ isotrivial? Are the $f_{i}$ isotrivial? Is $X$ rationally dominated by a product $X_{1} \times X_{2} \times X_{3}$, where $X_{i}$ dominates and is generically finite over $A_{i}$ ? We are inclined to think that the answers to all these questions should be affirmative, but we were only able to go further in the case where the $A_{i}$ are all elliptic curves.

\section{The 3-DIMEnSIONAL CASE}

We now come to our main result, which completely describes all smooth projective threefolds $X$ of maximal Albanese dimension and of general type, with $\chi\left(X, \omega_{X}\right)=0$.

Theorem 5.1. Let $X$ be a smooth projective threefold of maximal Albanese dimension and of general type, with $\chi\left(X, \omega_{X}\right)=0$.

There exist elliptic curves $E_{1}, E_{2}$, and $E_{3}$, double coverings $C_{j} \rightarrow E_{j}$ with associated involutions $\iota_{j}$, and a commutative diagram

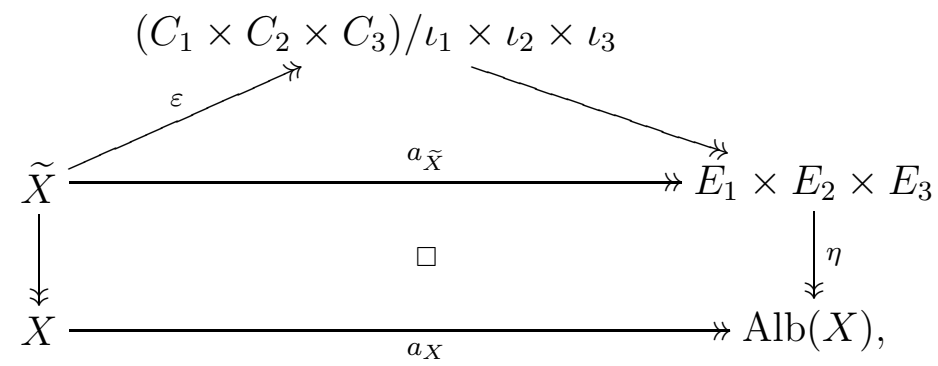

where $\eta$ is an isogeny and $\varepsilon$ a desingularization.

In other words, up to abelian étale covers, the Ein-Lazarsfeld examples (Example 4.1) are the only ones (in dimension 3 )! Note also that $a_{X}$ is not finite, but that it is finite on the canonical model of $X$.

Corollary 5.2. Under the hypotheses of the theorem, the Albanese mapping of $X$ is birationally a $(\mathbf{Z} / 2 \mathbf{Z})^{2}$-covering.

Proof. With the notation of Example 4.1, we set $A:=E_{1} \times E_{2} \times E_{3}$ and $\widetilde{X}_{i}:=\operatorname{Spec}\left(\mathscr{O}_{A} \oplus L_{i}^{\vee}\right)$, so that $f$ factors through the double coverings $\widetilde{f}_{i}:=\widetilde{X}_{i} \rightarrow A$. Since the action of $\operatorname{Ker}(\eta)$ on $A$ by translations lifts to $\widetilde{X}$, it leaves

$$
a_{\widetilde{X} *} \mathscr{O}_{\widetilde{X}} \simeq \mathscr{O}_{A} \oplus\left(L_{1}^{\vee} \otimes L_{2}^{\vee}\right) \oplus\left(L_{3}^{\vee} \otimes L_{1}^{\vee}\right) \oplus\left(L_{2}^{\vee} \otimes L_{3}^{\vee}\right)
$$


invariant, hence also each $L_{i}^{\vee}$. It follows that this action lifts to each $\widetilde{X}_{i}$, hence $\widetilde{f}_{i}$ descends to a double covering $X_{i} \rightarrow \operatorname{Alb}(X)$ through which $a_{X}$ factors.

Proof of the theorem. By Proposition 4.5.a), $a_{X}$ is surjective and $q(X)=3$ (see also [CH2], Corollary 4.3).

Moreover, by Corollary [3.5.b), $\operatorname{Alb}(X)$ is isogeneous to the product of three elliptic curves and, after passing to étale covers, we may assume that $a_{X}$ can be written as

$$
a_{X}: X \stackrel{\left(f_{1}, f_{2}, f_{3}\right)}{\longrightarrow} E_{1} \times E_{2} \times E_{3},
$$

where each $f_{i}: X \rightarrow E_{i}$ is a fibration, and that $\widehat{E}_{1} \times \widehat{E}_{2} \times\{0\}, \widehat{E}_{1} \times\{0\} \times \widehat{E}_{3}$, and $\{0\} \times \widehat{E}_{2} \times \widehat{E}_{3}$ are irreducible components of $V_{0}\left(\omega_{X}, a_{X}\right)$ (Proposition 4.3. b)).

Let $\{i, j, k\}=\{1,2,3\}$. As in $\$ 4$, we have a commutative diagram (15), where each $S_{i}$ is a smooth minimal surface of general type and $A_{i}=E_{i}$. The proof of the theorem is very long, so we will divide it in several steps. The general scheme of proof goes as follows:

- In the diagram (5), the fibrations $h_{i j}: S_{i} \rightarrow E_{j}$ are all isotrivial (Step 1); we let $C_{i j}$ be a (constant) general fiber.

- There exist finite groups $G_{i}$ acting on $C_{i j}$ such that $C_{i j} / G_{i} \simeq E_{j}$ and $C_{i k} / G_{i} \simeq E_{k}$, the surface $S_{i}$ is birational to $\left(C_{i j} \times C_{i k}\right) / G_{i}$, and $h_{i j}$ and $h_{i k}$ are the two projections (Step 2).

- At this point, it is quite easy to show that $X$ dominates a threefold $Y$ which is dominated by a product of 3 curves (Step 3).

- Taking an étale cover of $X$, we may assume that all the irreducible components of $V_{0}\left(\omega_{X}, a_{X}\right)$ pass through 0 . We then show (Step 4) that $V_{0}\left(\omega_{X}, a_{X}\right)$ has the same form as the corresponding locus of an Ein-Lazarsfeld threefold (see (3)), from which we deduce that $X$ is birationally isomorphic to $Y$ (Step 5) hence is also dominated by a product of 3 curves.

- Using the fact that $V_{0}\left(\omega_{X}, a_{X}\right)$ has no "extra" components, we finish the proof by showing that the groups $G_{i}$ all have order 2 (Step 6).

Step 1. The fibrations $h_{i j}: S_{i} \rightarrow E_{j}$ are all isotrivial.

We will denote by $C_{i j}$ a general (constant) fiber of $h_{i j}$.

Proof. By the semi-stable reduction theorem ([KKMS], Chapter II), there exist a finite cover $h: C \rightarrow E_{j}$, where $C$ is a smooth curve and commutative diagrams (for each $\alpha \in\{i, k\}$ )

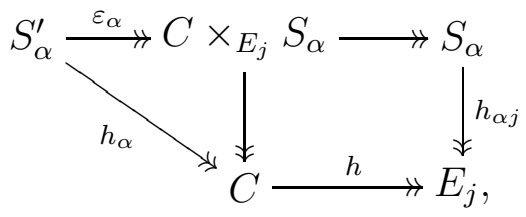

where $\varepsilon_{\alpha}$ is a modification and the fibers of $h_{\alpha}$ are all reduced connected curves, with nonsingular components crossing transversally. 
We also make a modification $\tau: X^{\prime} \rightarrow C \times_{E_{j}} X$ such that there exists a commutative diagram of morphisms between smooth varieties:

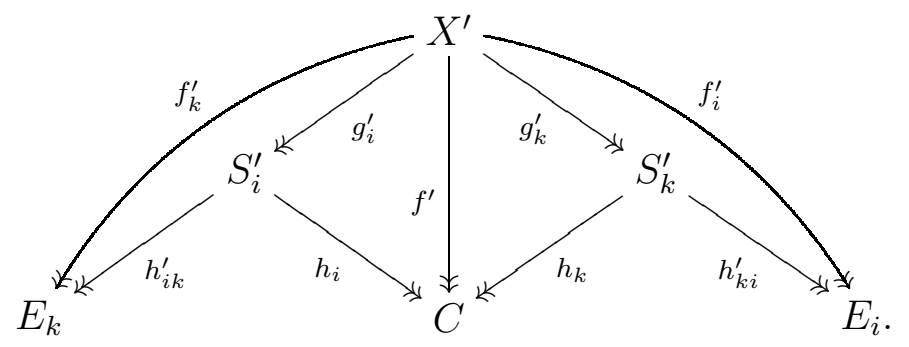

Let $\xi_{\alpha} \in \widehat{E}_{\alpha}$. By [V], Lemma 3.1] we have an inclusion

$$
f_{*}^{\prime}\left(\omega_{X^{\prime} / C} \otimes f_{i}^{\prime *} P_{\xi_{i}} \otimes f_{k}^{\prime *} P_{\xi_{k}}\right) \subseteq h^{*} f_{j *}\left(\omega_{X} \otimes f_{i}^{*} P_{\xi_{i}} \otimes f_{k}^{*} P_{\xi_{k}}\right)
$$

of locally free sheaves on $C$. Since $h_{\alpha}$ is flat with irreducible general fibers, $S_{i}^{\prime} \times_{C} S_{k}^{\prime}$ is irreducible, and we have a surjective morphism

$$
g_{i k}^{\prime}: X^{\prime} \stackrel{\left(g_{i}^{\prime}, g_{k}^{\prime}\right)}{\longrightarrow} S_{i}^{\prime} \times_{C} S_{k}^{\prime} .
$$

Moreover, $h_{i}$ and $h_{k}$ are semistable, hence by [AK], Proposition $6.4, S_{i}^{\prime} \times_{C} S_{k}^{\prime}$ has only rational Gorenstein singularities. After further modification of $X^{\prime}$, we may assume that $g_{i k}^{\prime}$ factors through a desingularization of $S_{i}^{\prime} \times_{C} S_{k}^{\prime}$ :

$$
g_{i k}^{\prime}: X^{\prime} \rightarrow Y_{i k}^{\prime} \stackrel{\varepsilon}{\rightarrow} S_{i}^{\prime} \times_{C} S_{k}^{\prime} .
$$

By definition of rational Gorenstein singularities, we have $\varepsilon_{*} \omega_{Y_{i k}^{\prime}}=\omega_{S_{i}^{\prime} \times_{C} S_{k}^{\prime}}$. Since $\omega_{X^{\prime} / Y_{i k}^{\prime}}$ is effective, we obtain an inclusion

$$
p_{i}^{*}\left(\omega_{S_{i}^{\prime} / C} \otimes h_{i k}^{\prime *} P_{\xi_{k}}\right) \otimes p_{k}^{*}\left(\omega_{S_{k}^{\prime} / C} \otimes h_{k i}^{\prime *} P_{\xi_{i}}\right) \subseteq g_{i k *}^{\prime}\left(\omega_{X^{\prime} / C} \otimes f_{i}^{\prime *} P_{\xi_{i}} \otimes f_{k}^{\prime *} P_{\xi_{k}}\right)
$$

of sheaves on $S_{i}^{\prime} \times_{C} S_{k}^{\prime}$. Pushing forward these sheaves to $C$, we obtain

$$
\begin{aligned}
h_{i *}\left(\omega_{S_{i}^{\prime} / C} \otimes h_{i k}^{\prime}{ }^{*} P_{\xi_{k}}\right) \otimes h_{k *}\left(\omega_{S_{k}^{\prime} / C} \otimes h_{k i}^{\prime}{ }^{*} P_{\xi_{i}}\right) & \subseteq f_{*}^{\prime}\left(\omega_{X^{\prime} / C} \otimes f_{i}^{\prime *} P_{\xi_{i}} \otimes f_{k}^{\prime *} P_{\xi_{k}}\right) \\
& \subseteq h^{*} f_{j *}\left(\omega_{X} \otimes f_{i}^{*} P_{\xi_{i}} \otimes f_{k}^{*} P_{\xi_{k}}\right),
\end{aligned}
$$

where the second inclusion comes from (6) $)$. Let $\{\alpha, \beta\}=\{i, k\}$. Both sheaves $h_{\alpha *}\left(\omega_{S_{\alpha}^{\prime} / C} \otimes\right.$ $\left.h_{\alpha \beta}^{\prime}{ }^{*} P_{\xi_{\beta}}\right)$ are nef $\left([\mathrm{V}]\right.$, Corollary 3.6). On the other hand, for $\xi_{i}$ and $\xi_{k}$ general and torsion, the sheaf in (17) has degree 0 by Proposition 4.7, hence

$$
\operatorname{deg}\left(h_{\alpha *}\left(\omega_{S_{\alpha}^{\prime} / C} \otimes h_{\alpha \beta}^{\prime}{ }^{*} P_{\xi_{\beta}}\right)\right)=0 .
$$

By [K1], Corollary 10.15, both sheaves $R^{1} h_{\alpha *}\left(\omega_{S_{\alpha}^{\prime} / C} \otimes h_{\alpha \beta}^{\prime}{ }^{*} P_{\xi_{\beta}}\right)$ are torsion-free and generically 0 , hence 0.5 On the other hand, we have $R^{1} h_{\alpha *} \omega_{S_{\alpha}^{\prime} / C}=\mathscr{O}_{C}([\mathrm{~K} 2]$, Proposition 7.6) hence, by the Grothendieck-Riemann-Roch theorem,

$$
\left[\operatorname{ch}\left(h_{\alpha *}\left(\omega_{S_{\alpha}^{\prime} / C}\right)\right)-\operatorname{ch}\left(\mathscr{O}_{C}\right)\right] \operatorname{Td}(C)=\operatorname{ch}\left(h_{\alpha *}\left(\omega_{S_{\alpha}^{\prime} / C} \otimes h_{\alpha \beta}^{\prime}{ }^{*} P_{\xi_{\beta}}\right)\right) \operatorname{Td}(C)
$$

\footnotetext{
${ }^{4}$ This is proved there for $\xi_{i}=\xi_{k}=0$, but the same proof works in general.

${ }^{5}$ Note that up to this point, the proof works in the more general situation where $a_{X}$ is surjective and $\operatorname{Alb}(X)$ has a 1-dimensional simple factor $E_{1}$.
} 
in the ring of cycles modulo numerical equivalence on $C$. This implies $\operatorname{deg}\left(h_{\alpha *} \omega_{S_{\alpha}^{\prime} / C}\right)=0$, and $h_{\alpha}$ is locally trivial (see e.g., [BPV], Theorem III.17.3). Hence $h_{\alpha j}$ is isotrivial (for each $\alpha \in\{i, k\})$.

Step 2. There exist finite groups $G_{i}$ acting on $C_{i j}$ such that $C_{i j} / G_{i} \simeq E_{j}$ and $C_{i k} / G_{i} \simeq E_{k}$, the surface $S_{i}$ is birational to the quotient $\left(C_{i j} \times C_{i k}\right) / G_{i}$ for the diagonal action of $G_{i}$, and $h_{i j}$ and $h_{i k}$ are identified with the two projections.

This is a consequence of the following (probably classical) result.

Lemma 5.3. Let $S$ be a smooth projective surface with an isotrivial fibration $h_{1}: S \rightarrow \Gamma_{1}$ onto an irrational curve with (constant) irrational general fiber $F_{1}$.

a) There exist a smooth curve $F_{2}$ and a finite group $H$ acting faithfully on $F_{1}$ and $F_{2}$ such that $\Gamma_{1}$ is isomorphic to $F_{2} / H$, the surface $S$ is birationally isomorphic to the diagonal quotient $\left(F_{1} \times F_{2}\right) / H$, and $h_{1}$ is the composition $S \stackrel{\sim}{\sim}\left(F_{1} \times F_{2}\right) / H \rightarrow F_{2} / H \simeq \Gamma_{1}$. Let $h_{2}$ be the composition $S \stackrel{\sim}{\sim} \rightarrow\left(F_{1} \times F_{2}\right) / H \rightarrow F_{1} / H$.

b) Assume $S$ is of general type. Any isotrivial fibration $h: S \rightarrow \Gamma$ onto an irrational curve $\Gamma$ is either $h_{1}$ or $h_{2}$ followed by an isomorphism between $F_{1} / H$ or $F_{2} / H$ with $\Gamma$.

Proof. Item a) is well-known and can be found in [S]. Let us prove b). Since $\Gamma$ is irrational, $h$ induces an isotrivial fibration $h^{\prime}:\left(F_{1} \times F_{2}\right) / H \rightarrow \Gamma$. Let $D_{2}$ be a general (constant irrational) fiber of $h^{\prime}$. The quotient map $\pi: F_{1} \times F_{2} \rightarrow\left(F_{1} \times F_{2}\right) / H$ is étale outside a finite set. Hence the Stein factorization $g$ of $h^{\prime} \circ \pi$ in the diagram

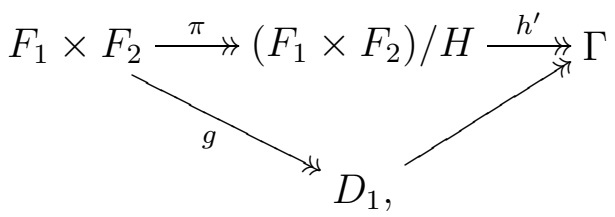

is also isotrivial, with general fiber $D_{2}^{\prime}$ a (fixed) étale cover of $D_{2}$. By a), there is a base change $D_{1}^{\prime} \rightarrow D_{1}$ and a surjective morphism $t=\left(t_{1}, t_{2}\right): D_{1}^{\prime} \times D_{2}^{\prime} \rightarrow F_{1} \times F_{2}$. Since $S$ is of general type, $F_{1}$ and $F_{2}$ are each of genus $\geq 2$, hence each $t_{i}$ must factor through one of the projections $p_{j}: D_{1}^{\prime} \times D_{2}^{\prime} \rightarrow D_{j}^{\prime}$.

If $h$ factors through neither $h_{1}$ nor $h_{2}$, the curve $D_{2}^{\prime}$ dominates both $F_{1}$ and $F_{2}$, hence $t_{1}$ and $t_{2}$ cannot factor through $p_{1}$. Thus they must factor through $p_{2}$, which contradicts the fact that $t$ is surjective.

Let now $Y_{j}$ be a resolution of singularities of the irreducible threefold $S_{i} \times_{E_{j}} S_{k}$ and let $Y$ be a resolution of singularities of the component of $Y_{1} \times_{E_{2} \times E_{3}} S_{1}$ that dominates both $Y_{1}$ 
and $Y_{3}$. After modification of $X$, we obtain a diagram

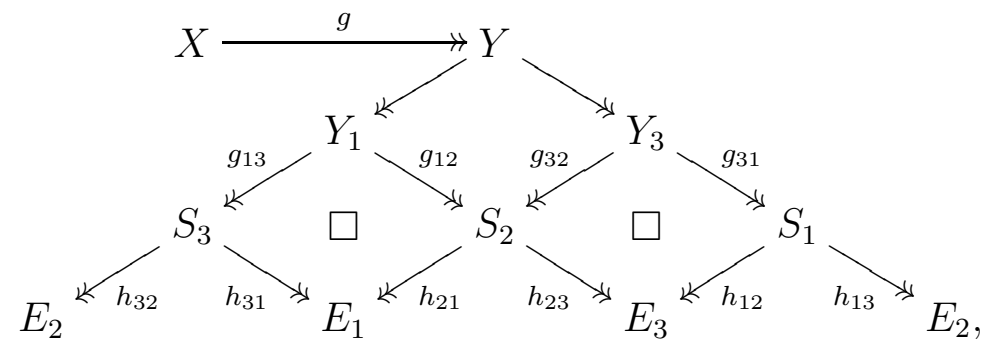

where the squares are birationally cartesian and the isotrivial morphisms $h_{i j}: S_{i} \rightarrow E_{j}$ fit into diagrams

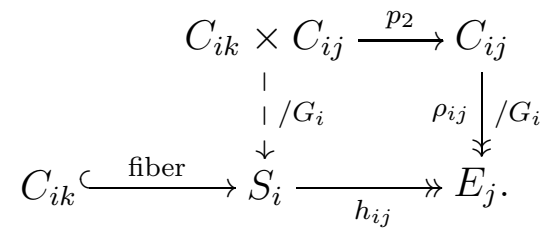

Step 3. The threefold $Y$ is dominated by a product of three curves.

The dominant maps $C_{31} \times C_{32} \rightarrow S_{3}$ and $C_{21} \times C_{23} \rightarrow S_{2}$ induce a factorization

$$
\left(\left(\rho_{31}, \rho_{21}\right), \rho_{32}, \rho_{23}\right):\left(C_{31} \times_{E_{1}} C_{21}\right) \times C_{32} \times C_{23}-\rightarrow S_{3} \times_{E_{1}} S_{2} \rightarrow E_{1} \times E_{2} \times E_{3} .
$$

The (Stein factorization of the) morphism $Y_{1} \rightarrow E_{2} \times E_{3}$ is therefore isotrivial (its fibers are dominated by the curve $\left.C_{31} \times_{E_{1}} C_{21}\right)$. Thus, $Y$ is dominated by the product

$$
\left(C_{31} \times_{E_{1}} C_{21}\right) \times\left(C_{12} \times_{E_{2}} C_{32}\right) \times\left(C_{23} \times_{E_{3}} C_{13}\right)
$$

of three (possibily reducible) curves.

Going back to the proof of Theorem 5.1, after passing to an étale cover, we may and will assume, from now on, that the following holds (\$2.2):

$$
\text { All the irreducible components of } V_{0}\left(\omega_{X}, a_{X}\right) \text { pass through } 0 .
$$

One checks, using $92.1,92.2$, and Proposition 4.5. $\mathrm{d}$ ), that if we want $X$ to be birationally covered by a product $\Gamma_{1} \times \Gamma_{2} \times \Gamma_{3}$, with morphisms $\Gamma_{i} \rightarrow E_{i}$, as in the conclusion of the theorem, we must have the following.

Step 4. We have

$$
V_{0}\left(\omega_{X}, a_{X}\right)=\left(\widehat{E}_{1} \times \widehat{E}_{2} \times\{0\}\right) \cup\left(\widehat{E}_{1} \times\{0\} \times \widehat{E}_{3}\right) \cup\left(\{0\} \times \widehat{E}_{2} \times \widehat{E}_{3}\right) .
$$

We already know that $V_{0}\left(\omega_{X}, a_{X}\right)$ contains the right-hand-side (Proposition 4.3. b)) and we must prove that it has no other components.

Proof. Assume $V_{0}\left(\omega_{X}, a_{X}\right)$ has another component $\widehat{T}$. It has dimension 2 (Corollary 3.5.a)) and, after possibly permuting the indices, we may assume the neutral component $\widehat{E}_{1}^{\prime}$ of $\widehat{T} \cap\left(\widehat{E}_{1} \times \widehat{E}_{2} \times\{0\}\right)$ is neither $\widehat{E}_{1} \times\{0\} \times\{0\}$ nor $\{0\} \times \widehat{E}_{2} \times\{0\}$. This yields an elliptic curve $E_{1}^{\prime}$ which is a quotient of $E_{1} \times E_{2}$ which does not factor through either projection. As 
we saw right before Proposition 4.7, the induced map $f_{4}: X \rightarrow E_{1}^{\prime}$ is a fibration. It factors as

$$
f_{4}: X \longrightarrow S_{3} \stackrel{h_{34}}{\longrightarrow} E_{1}^{\prime}
$$

where, by Step 1, $h_{34}$ is isotrivial. By Lemma 5.3.b), $h_{34}$ must factor through one of the projections $h_{31}: S_{3} \rightarrow E_{1}$ or $h_{32}: S_{3} \rightarrow E_{2}$ so we reach a contradiction.

Step 5. The morphism $g: X \rightarrow Y$ is birational.

Proof. Consider, in the diagram (8), the generically finite morphism $v_{1}: X \rightarrow Y_{1}$ and the three fibrations $f_{\alpha}^{\prime}: Y_{1} \rightarrow E_{\alpha}$, for $\alpha \in\{1,2,3\}$. Since $X, Y_{1}$, and $S_{3}$ are all of maximal Albanese dimensions, $\omega_{X / Y_{1}}$ and $\omega_{Y_{1} / S_{3}}$ are effective, hence

$$
h^{0}\left(X, \omega_{X} \otimes a_{X}^{*} P_{\xi}\right) \geq h^{0}\left(Y_{1}, \omega_{Y_{1}} \otimes\left(f_{1}^{\prime}, f_{2}^{\prime}\right)^{*} P_{\xi}\right) \geq h^{0}\left(S_{3}, \omega_{S_{3}} \otimes\left(h_{31}, h_{32}\right)^{*} P_{\xi}\right)
$$

for all $\xi \in \widehat{E}_{1} \times \widehat{E}_{2}$. Moreover, for $\xi$ non-zero, we have by Proposition 4.5.d)

$$
h^{2}\left(X, \omega_{X} \otimes a_{X}^{*} P_{\xi}\right)=h^{3}\left(X, \omega_{X} \otimes a_{X}^{*} P_{\xi}\right)=0
$$

hence, since $\chi\left(X, \mathscr{O}_{X}\right)=0$,

$$
h^{0}\left(X, \omega_{X} \otimes a_{X}^{*} P_{\xi}\right)=h^{1}\left(X, \omega_{X} \otimes a_{X}^{*} P_{\xi}\right) .
$$

Finally, for $\xi$ general in $\widehat{E}_{1} \times \widehat{E}_{2}$, we have, as in the proof of Theorem 3.1 , since $g_{3}: X \rightarrow S_{3}$ has connected fibers,

$$
h^{1}\left(X, \omega_{X} \otimes a_{X}^{*} P_{\xi}\right)=h^{0}\left(S_{3}, \omega_{S_{3}} \otimes\left(h_{31}, h_{32}\right)^{*} P_{\xi}\right) .
$$

Therefore, for $\xi \in \widehat{E}_{1} \times \widehat{E}_{2}$ general, we obtain

$$
h^{0}\left(X, \omega_{X} \otimes a_{X}^{*} P_{\xi}\right)=h^{0}\left(Y_{1}, \omega_{Y_{1}} \otimes\left(f_{1}^{\prime}, f_{2}^{\prime}\right)^{*} P_{\xi}\right) .
$$

The induced morphism $Y \rightarrow E_{1} \times E_{2} \times E_{3}$ is the Albanese mapping of $Y$. Since in any event, we always have

$$
h^{0}\left(X, \omega_{X} \otimes a_{X}^{*} P_{\xi}\right) \geq h^{0}\left(Y, \omega_{Y} \otimes a_{Y}^{*} P_{\xi}\right) \geq h^{0}\left(Y_{1}, \omega_{Y_{1}} \otimes\left(f_{1}^{\prime}, f_{2}^{\prime}\right)^{*} P_{\xi}\right)
$$

for all $\xi \in \widehat{E}_{1} \times \widehat{E}_{2}$, we obtain

$$
h^{0}\left(X, \omega_{X} \otimes a_{X}^{*} P_{\xi}\right)=h^{0}\left(Y, \omega_{Y} \otimes a_{Y}^{*} P_{\xi}\right)
$$

for $\xi$ general in $\widehat{E}_{1} \times \widehat{E}_{2}$, hence also, by Step 3 , for $\xi$ general in $V_{0}\left(\omega_{X}, a_{X}\right)$. But for $\xi \notin$ $V_{0}\left(\omega_{X}, a_{X}\right)$, both sides of (11) vanish. By Lemma 5.4 below, we conclude that $g$ is a birational morphism.

The following lemma (used in the proof above) is in the spirit of [HP], Theorem 3.1.

Lemma 5.4. Let $X \stackrel{g}{\rightarrow} Y \stackrel{f}{\rightarrow} A$ be generically finite morphisms between smooth projective threefolds, where $A$ is an abelian threefold, such that $f$ and $f \circ g$ are both minimal. Assume that $X$ is of general type with $\chi\left(X, \omega_{X}\right)=0$ and that there exists an open subset $U \subseteq \widehat{A}$ with $\operatorname{codim}_{\widehat{A}}(\widehat{A}-U) \geq 2$ such that

$$
h^{0}\left(X, \omega_{X} \otimes g^{*} f^{*} P_{\xi}\right)=h^{0}\left(Y, \omega_{Y} \otimes f^{*} P_{\xi}\right)
$$

for all $\xi \in U$. Then $g$ is birational. 
Proof. By \$2.2, we can write $g_{*} \omega_{X} \simeq \omega_{Y} \oplus \mathscr{E}$, and we need to show that the sheaf $\mathscr{E}$ is zero. Since $\mathscr{E}$ is torsion-free and $f$ is generically finite, it is sufficient to prove $f_{*} \mathscr{E}=0$.

As we saw at the beginning of $\$ 5$, we have $q(X)=3$, hence $f \circ g$ is the Albanese mapping of $X$. By Proposition 4.5, d), for each $i \in\{2,3\}$, we then have $\{0\}=V_{i}\left(\omega_{X}, f \circ g\right)=$ $V_{i}\left(g_{*} \omega_{X}, f\right)$, hence $V_{i}\left(f_{*} \mathscr{E}\right) \subseteq\{0\}$.

Since $q(X)=3$, we also have $q(Y)=3$, hence $h^{i}\left(Y, g_{*} \omega_{X}\right)=h^{i}\left(Y, \omega_{Y}\right)$. It follows that $V_{i}\left(f_{*} \mathscr{E}\right)$ is empty.

The assumption $\chi\left(X, \omega_{X}\right)=0$ implies $\chi\left(Y, \omega_{Y}\right)=0$ by (1). Thus,

$$
V_{0}\left(f_{*} \mathscr{E}\right)=V_{1}\left(f_{*} \mathscr{E}\right) \subseteq \widehat{A}-U
$$

Since $\operatorname{codim}_{\widehat{A}}(\widehat{A}-U)>1$, the sheaf $f_{*} \mathscr{E}$ is therefore M-regular in the sense of [PP], Definition 2.1 (see also Remark 2.3), hence continuously globally generated ([PP], Definition 2.10 and Proposition 2.13). Since $H^{0}\left(A, f_{*} \mathscr{E} \otimes P_{\xi}\right)=0$ for all $\xi \in U$, we obtain $f_{*} \mathscr{E}=0$.

Let us summarize what we know. Let $\{i, j, k\}=\{1,2,3\}$. The curve $C_{i j}$ is the (constant) general fiber of the isotrivial fibration $S_{i} \rightarrow E_{k}$; it is acted on by a group $G_{i}$ and $C_{i j} / G_{i} \simeq E_{j}$ (Step 2). The fibration $g_{i}: X \rightarrow S_{i}$ is also isotrivial; as we saw in Step 3, its general fiber $C_{i}$ is dominated by the curve $C_{j i} \times_{E_{i}} C_{k i}$ but also maps onto $C_{j i}$ and $C_{k i}$. Finally, a general fiber $F_{k}$ of the isotrivial fibration $f_{k}: X \rightarrow E_{k}$ is an isotrivial fibration over $C_{i j}$ with (constant) general fiber $C_{i}$. The situation is summarized in the following diagram:

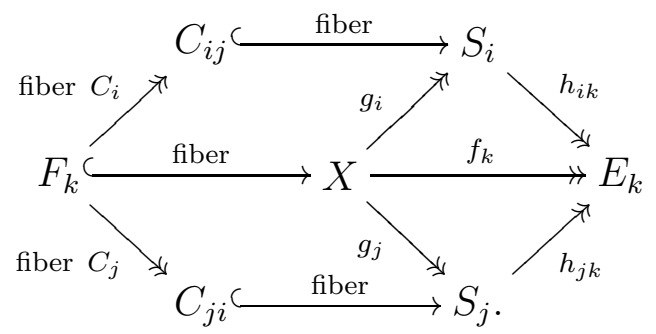

By Lemma 5.3, there exists a finite group $H_{k}$ acting faithfully on $C_{i}$ and $C_{j}$ such that $C_{i j} \simeq C_{j} / H_{k}, C_{j i} \simeq C_{i} / H_{k}$, and $F_{k}$ is isomorphic to the diagonal quotient $\left(C_{i} \times C_{j}\right) / H_{k}$. Moreover, the maps to $C_{i j}$ and $C_{j i}$ are the natural projections. So we have diagrams

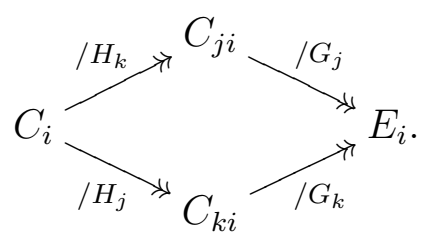

Let $D_{1}$ be the Galois closure of $C_{1}$ over $E_{1}$ and set $G=\operatorname{Gal}\left(D_{1} / E_{1}\right)$. Let $\{j, k\}=\{2,3\}$. There is a normal subgroup $N_{j} \triangleleft G$ such that $G_{j}=G / N_{j}$ and $G$ acts on $C_{j k}$ via this quotient. By Step 2, the surface $S_{j}$ is birationally isomorphic to $\left(C_{j 1} \times C_{j k}\right) / G_{j}$, hence to $\left(D_{1} \times C_{j k}\right) / G$. Therefore, the modification $Y_{1}$ of $S_{2} \times{ }_{E_{1}} S_{3}$ (see (8) ) is birationally isomorphic to $\left(D_{1} \times C_{23} \times C_{32}\right) / G$.

Step 6. The group $G$ is isomorphic to $\mathbf{Z} / 2 \mathbf{Z}$. 
We begin with a lemma which is probably well-known. We denote by $\operatorname{Irr}(G)$ the set of isomorphism classes of irreducible representations of $G$.

Lemma 5.5. Let $E$ be an elliptic curve and let $\pi: D \rightarrow E$ be a Galois cover with group $G$. We can write

$$
\pi_{*} \mathscr{O}_{D}=\bigoplus_{\chi \in \operatorname{Irr}(G)} \bigoplus_{i} \mathscr{V}_{\chi, i}
$$

where each vector bundle $\mathscr{V}_{\chi, i}$ is semistable and $G$-invariant, and the representation of $G$ on the general fiber of each $\mathscr{V}_{\chi, i}$ is a direct sum of $\chi$. Moreover, for each $\chi \neq 1$, the dual vector bundle $\mathscr{V}_{\chi, i}^{\vee}$ is either ample or a direct sum of non-zero torsion line bundles.

Proof. The groups $G$ acts on $\pi_{*} \mathscr{O}_{D}$. Identifying each representation $\chi$ with its character, we consider the endomorphism $\sum_{g \in G} \chi(g) g$ of $\pi_{*} \mathscr{O}_{D}$ and we denote by $\mathscr{V}_{\chi}^{\vee}$ its image. We then have

$$
\pi_{*} \mathscr{O}_{D}=\bigoplus_{\chi} \mathscr{N}_{\chi}
$$

where the general fiber of $\mathscr{V}_{\chi}$ is a (nonzero) direct sum of $\chi$ as a $G$-module.

The Harder-Narasimhan filtration

$$
0=\mathscr{V}_{\chi}^{\ell} \subseteq \mathscr{V}_{\chi}^{\ell-1} \subseteq \cdots \subseteq \mathscr{V}_{\chi}^{0}=\mathscr{V}_{\chi}
$$

is preserved by the $G$-action. Serre duality shows that since we are on an elliptic curve, all the corresponding extensions are trivial, hence $\mathscr{V}_{\chi}$ is the direct sum of the $G$-invariant semistable bundles $\mathscr{V}_{\chi, i}=\mathscr{V}_{\chi}^{i} / \mathscr{V}_{\chi}^{i+1}$, for $0 \leq i<\ell$.

As a direct summand of $\pi_{*} \omega_{D}=\left(\pi_{*} \mathscr{O}_{D}\right)^{\vee}$, each vector bundle $\mathscr{V}_{\chi, i}^{\vee}$ is nef $([\mathrm{V}]$, Corollary 3.6). Moreover, it is ample if it has positive degree. Consider the maximal degree-0 subsheaf $\mathscr{F}$ of $\pi_{*} \mathscr{O}_{D}$, i.e., the direct sum of all $\mathscr{V}_{\chi, i}$ that have degree 0 . By $[\mathrm{KP}]$, Lemma 3.2 and $3.4, \mathscr{F}$ is a $G$-invariant subalgebra and induces an étale cover of $E$, hence is a direct sum of torsion line bundles.

Let us continue with the Galois cover $\pi: D \rightarrow E$ with group $G$ as in the lemma and assume moreover that for each $j \in\{2,3\}$, we have a Galois cover $\pi_{j}: D_{j} \rightarrow E_{j}$ with Galois group $G_{j}=G / N_{j}$, where $g\left(D_{j}\right) \geq 2$ and $E_{j}$ is an elliptic curve.

Then $G$ acts on $D_{1} \times D_{2} \times D_{3}$ diagonally. Let $Z$ be the quotient, let $\varepsilon: Y \rightarrow Z$ be a resolution, and consider

$$
t: Y \stackrel{\varepsilon}{\rightarrow} Z \rightarrow E_{1} \times E_{2} \times E_{3} .
$$

Lemma 5.6. Assume

$$
V_{0}\left(\omega_{Y}, t\right) \subseteq\left(\widehat{E}_{1} \times \widehat{E}_{2} \times\{0\}\right) \cup\left(\widehat{E}_{1} \times\{0\} \times \widehat{E}_{3}\right) \cup\left(\{0\} \times \widehat{E}_{2} \times \widehat{E}_{3}\right)
$$

and $V_{2}\left(\omega_{Y}, t\right) \cup V_{3}\left(\omega_{Y}, t\right) \subseteq\{0\}$. Then $N_{2}=N_{3}$ and $G_{2} \simeq G_{3} \simeq \mathbf{Z} / 2 \mathbf{Z}$.

Proof. We decompose $\pi_{*} \mathscr{O}_{D}$ as in (13) and write similarly

$$
\pi_{j *} \mathscr{O}_{D_{j}}=\bigoplus_{\mu \in \operatorname{Irr}\left(G_{j}\right)} \bigoplus_{i} \mathscr{V}_{\mu, i}^{j}
$$


Since quotient singularities are rational, we have as in Example 4.1

$$
t_{*} \omega_{Y} \simeq\left(q_{*} \mathscr{O}_{Z}\right)^{\vee} \simeq\left(( \pi _ { * } \mathscr { O } _ { D } ) ^ { \vee } \otimes \left(\left(\pi_{2 *} \mathscr{O}_{D_{2}}\right)^{\vee} \otimes\left(\left(\pi_{3 *} \mathscr{O}_{D_{3}}\right)^{\vee}\right)^{G}\right.\right.
$$

Let $\mu$ be an non-trivial element of $\operatorname{Irr}\left(G_{2}\right)$. Since $G_{2}$ is a quotient of $G$, the representation $\mu$ and its complex conjugate $\bar{\mu}$ are also in $\operatorname{Irr}(G)$. Then, the vector bundle

$$
\mathscr{G}:=\left(\mathscr{V}_{\bar{\mu}, 1}^{\vee} \otimes \mathscr{V}_{\mu, 1}^{2 \vee} \otimes \mathscr{O}_{E_{3}}\right)^{G}
$$

on $E_{1} \times E_{2} \times E_{3}$ is a non-zero direct summand of both $\mathscr{V}_{\bar{\mu}, 1}^{\vee} \otimes \mathscr{V}_{\mu, 1}^{2 \vee} \otimes \mathscr{O}_{E_{3}}$ and $t_{*} \omega_{Y}$.

Assume that $\mathscr{V}_{\mu, 1}^{2}$ has degree 0 , hence is a direct sum of non-trivial torsion line bundles.

- If $\operatorname{deg}\left(\mathscr{V}_{\bar{\mu}, 1}^{\vee}\right)=0$, the sheaf $\mathscr{G}$ is a direct sum of non-trivial torsion line bundles, which is impossible since $V_{3}(\mathscr{G}) \subseteq V_{3}\left(\omega_{Y}, t\right)=\{0\}$.

- If $\mathscr{V}_{\bar{\mu}, 1}^{\vee}$ is ample, we can write

$$
\mathscr{G}=\bigoplus_{k}\left(\mathscr{G}_{k} \otimes P_{\xi_{k}} \otimes \mathscr{O}_{E_{3}}\right)
$$

where $\mathscr{G}_{k}$ is a direct summand of $\mathscr{V}_{\bar{\mu}, 1}^{\vee}$, hence ample, and the $\xi_{k}$ are non-zero torsion points in $\widehat{E}_{2}$. This is again impossible, because $V_{2}(\mathscr{G}) \subseteq V_{2}\left(\omega_{Y}, t\right)=\{0\}$.

Therefore, $\mathscr{V}_{\mu, 1}^{j \vee}$ is ample for all $\mu$ non-trivial in $\operatorname{Irr}\left(G_{j}\right)$.

If $\operatorname{Card}\left(G_{2}\right)>2$, or if $N_{2} \neq N_{3}$, we may take non-trivial $\chi \in \operatorname{Irr}(G), \mu \in \operatorname{Irr}\left(G_{2}\right)$, and $\nu \in \operatorname{Irr}\left(G_{3}\right)$ such that $\chi$ is a subrepresentation of $\mu \otimes \nu$. The vector bundle

$$
\mathscr{H}:=\left(\mathscr{V}_{\bar{\chi}, 1}^{\vee} \otimes \mathscr{V}_{\mu, 1}^{2 \vee} \otimes \mathscr{V}_{\nu, 1}^{3 \vee}\right)^{G}
$$

is then non-zero and a direct summand of $t_{*} \omega_{Y}$ (and $\mathscr{V}_{\mu, 1}^{2 \vee}$ and $\mathscr{V}_{\nu, 1}^{3 \vee}$ are ample).

If $\mathscr{V}_{\bar{\chi}, 1}^{\vee}$ is ample, since $\mathscr{H}$ is a direct summand of $\mathscr{V}_{\bar{\chi}, 1}^{\vee} \otimes \mathscr{V}_{\mu, 1}^{2 \vee} \otimes \mathscr{V}_{\nu, 1}^{3 \vee}$, we have $V_{m}(\mathscr{H})=$ $\varnothing$ for all $m \in\{1,2,3\}$. Hence $h^{0}\left(E_{1} \times E_{2} \times E_{3}, \mathscr{H} \otimes P_{\xi}\right)$ is a non-zero constant for all $\xi \in \widehat{E}_{1} \times \widehat{E}_{2} \times \widehat{E}_{3}$ and $V_{0}(\mathscr{H})=\widehat{E}_{1} \times \widehat{E}_{2} \times \widehat{E}_{3}$, which contradicts our assumptions.

If $\mathscr{V}_{\bar{\chi}, 1}^{\vee}$ is a direct sum of non-trivial torsion line bundles, we may write

$$
\mathscr{H}=\bigoplus_{k}\left(P_{\xi_{k}} \otimes \mathscr{H}_{k}\right)
$$

where the $\xi_{k}$ are non-zero torsion points in $\widehat{E}_{1}$ and $\mathscr{H}_{k}$ is a direct summand of $\mathscr{V}_{\mu, 1}^{2 \vee} \otimes$ $\mathscr{V}_{\nu, 1}^{3 \vee}$. Then $V_{0}(\mathscr{H})$, hence also $V_{0}\left(\omega_{Y}, t\right)$, contains $\left\{-\xi_{1}\right\} \times \widehat{E}_{2} \times \widehat{E}_{3}$, which contradicts our assumptions.

We now apply this second lemma to the Galois covers $\pi: D_{1} \rightarrow E_{1}, \pi_{2}: C_{23} \rightarrow E_{2}$, and $\pi_{3}: C_{32} \rightarrow E_{3}$. The variety $Y$ of the lemma is the variety $Y_{1}$ of the proof, and since $V_{0}\left(\omega_{Y_{1}}, t\right) \subseteq V_{0}\left(\omega_{X}, a_{X}\right)$ (see 2.2$)$, the hypotheses of the lemma are satisfied (Step 4).

We obtain $N_{2}=N_{3}$, hence the coverings $C_{j i} \rightarrow E_{i}$ and $C_{k i} \rightarrow E_{i}$ are the same (see (12)), and also $G / N_{j} \simeq \mathbf{Z} / 2 \mathbf{Z}$, so that they are double covers. Denote them by $C_{i}^{\prime} \rightarrow E_{i}$. By the proof of Step 3 (see (9) ) , $X$ is birational to $\left(C_{1}^{\prime} \times C_{2}^{\prime} \times C_{3}^{\prime}\right) /(\mathbf{Z} / 2 \mathbf{Z})$. Since the latter 
variety contains no rational curves, there is a birational morphism from $X$ to it. This finishes the proof of Theorem 5.1.

\section{VARIETIES With $P_{1}=1$}

It follows from [U] and 2.1.5 that varieties $X$ of maximal Albanese dimension and $P_{1}(X)=1$ satisfy $\chi\left(X, \omega_{X}\right)=0$. We presented in Example 4.2 a construction of Chen and Hacon of such a variety which is in addition of general type. We gather here some properties of these varieties (most of them taken from [U]).

Proposition 6.1. Let $X$ be a smooth projective variety of maximal Albanese dimension $n$, with $P_{1}(X)=1$.

a) We have an isomorphism

$$
a_{X}^{*}: \dot{\bigwedge} H^{0}\left(A, \Omega_{A}\right) \simeq H^{0}\left(X, \Omega_{X}^{\bullet}\right)
$$

In particular, $h^{j}\left(X, \mathscr{O}_{X}\right)=\left(\begin{array}{l}n \\ j\end{array}\right)$ for all $j$, hence $\chi\left(X, \omega_{X}\right)=0$, and the Albanese mapping $a_{X}: X \rightarrow \operatorname{Alb}(X)$ is surjective.

b) The point 0 is isolated in $V_{0}\left(\omega_{X}, a_{X}\right)$.

Proof. Replacing $X$ with a modification, we may assume that there is a factorization $a_{X}$ : $X \rightarrow Z \rightarrow \operatorname{Alb}(X)$, where $Z$ is a desingularization of $a_{X}(X)$, so that $P_{1}(Z) \leq P_{1}(X)=1$. It follows from [U] (or [M], Corollary (3.5)) that $a_{X}(X)$ is a translate of an abelian subvariety of $\operatorname{Alb}(X)$, hence $a_{X}$ is surjective. Item a) then follows from another result of Ueno $([\mathrm{U}]$, or [M], Corollary (3.4)).

By \2.2, we can write $a_{X *} \omega_{X} \simeq \omega_{A} \oplus \mathscr{E} \simeq \mathscr{O}_{A} \oplus \mathscr{E}$. The sheaf $\mathscr{E}$ then satisfies $V_{i}(\mathscr{E})-\{0\}=V_{i}\left(\omega_{X}, a_{X}\right)-\{0\}$ for all $i$. Since $1=P_{1}(X)=1+h^{0}(A, \mathscr{E})$, the point 0 is not in the closed set $V_{0}(\mathscr{E})$, hence is isolated in $V_{0}\left(\omega_{X}, a_{X}\right)$. This proves b).

Remark 6.2. Regarding item b), to be more precise, a smooth projective variety $X$ of maximal Albanese dimension satisfies $P_{1}(X)=1$ if and only if 0 is isolated in $V_{0}\left(\omega_{X}, a_{X}\right)$.

Theorem 6.3. Let $X$ be a smooth projective threefold of maximal Albanese dimension and of general type. If $P_{1}(X)=1$, the variety $X$ is a modification of an abelian étale cover of a Chen-Hacon threefold.

It is then very easy to describe all smooth projective threefolds $X$ of maximal Albanese dimension and of general type, with $P_{1}(X)=1$. Start from a Chen-Hacon threefold $Y$ as in Example 4.2, with Albanese mapping $a_{Y}: Y \rightarrow E_{1} \times E_{2} \times E_{3}$. It satisfies

$$
V_{0}\left(\omega_{Y}, a_{Y}\right)=\{0\} \cup\left(\widehat{E}_{1} \times \widehat{E}_{2} \times\left\{\xi_{3}\right\}\right) \cup\left(\widehat{E}_{1} \times\left\{\xi_{2}\right\} \times \widehat{E}_{3}\right) \cup\left(\left\{\xi_{1}\right\} \times \widehat{E}_{2} \times \widehat{E}_{3}\right),
$$

where each $\xi_{j} \in \widehat{E}_{j}$ has order 2. Take an isogeny $A \rightarrow E_{1} \times E_{2} \times E_{3}$ corresponding to a (finite) subgroup of $\widehat{E}_{1} \times \widehat{E}_{2} \times \widehat{E}_{3}$ which contains none of the points $\xi_{1}, \xi_{2}, \xi_{3}$. Finally, take for $X$ a modification of $Y \times_{E_{1} \times E_{2} \times E_{3}} A$. 
Proof of the theorem. Replacing $a_{X}$ with it Stein factorization, we will assume that $X$ is normal and $a_{X}$ is finite. By Theorem 5.1, there exist elliptic curves $E_{1}, E_{2}$, and $E_{3}$, double coverings $\rho_{i}: C_{i} \rightarrow E_{i}$, with involution $\iota_{i}$, and a commutative diagram

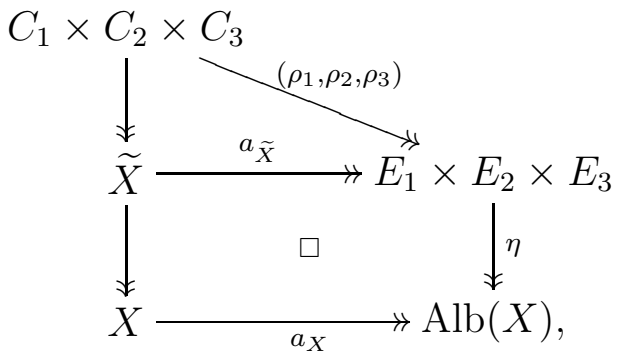

where $\eta$ is an isogeny (the variety $\widetilde{X}$ is the variety $Z$ of Example 4.1) and both $a_{X}$ and $a_{\widetilde{X}}$ are $(\mathbf{Z} / 2 \mathbf{Z})^{2}$-Galois coverings. In particular, $X$ has rational singularities.

We denote by $K$ the (finite) kernel of $\eta$ and by $K_{i}$ the image of the projection $K \rightarrow E_{i}$, so that $K$ is a subgroup of $\widetilde{K}:=K_{1} \times K_{2} \times K_{3}$. The elliptic curve $F_{i}:=E_{i} / K_{i}$ embeds in $\operatorname{Alb}(X)$; let $\pi_{i}: \operatorname{Alb}(X) \rightarrow A_{i}$ be the quotient. The natural morphism $h_{i}: X \rightarrow A_{i}$ is an isotrivial fibration and we denote by $D_{i}$ its general (constant) fiber. Now we consider the square restricted to fibers:

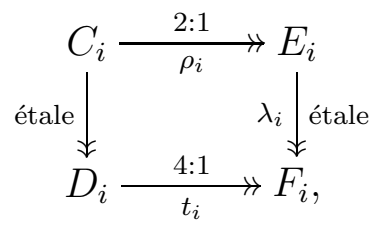

where $t_{i}$ is a $(\mathbf{Z} / 2 \mathbf{Z})^{2}$-cover. Since $D_{i} \times_{F_{i}} E_{i}$ is disconnected and factors as $C_{i} \sqcup C_{i} \rightarrow C_{i} \stackrel{\rho_{i}}{\rightarrow} E_{i}$, there is a non-zero 2-torsion point $\xi_{i} \in \widehat{F}_{i}$ such that $\xi_{i} \in \operatorname{Ker}\left(t_{i}^{*}\right) \cap \operatorname{Ker}\left(\lambda_{i}^{*}\right)$, the morphism $t_{i}$ factors as $D_{i} \stackrel{s_{i}}{\rightarrow} D_{i}^{\prime} \rightarrow F_{i}$, where $s_{i}$ is a double étale cover, and $C_{i} \simeq D_{i}^{\prime} \times_{F_{i}} E_{i}$. It follows that the group $K_{i}$ acts on $C_{i}$, and the involution $\iota_{i}$ and the $K_{i}$-action commute. Therefore, $\widetilde{K}$ acts on $C_{1} \times C_{2} \times C_{3}$ and this action commutes with the involution $\left(\iota_{1}, \iota_{2}, \iota_{3}\right)$. It follows that $\widetilde{K}$ acts on $\widetilde{X}$ and the Albanese mapping $a_{\widetilde{X}}$ is $\widetilde{K}$-equivariant. Set $Y:=\widetilde{X} / \widetilde{K}$.

Lemma 6.4. The quotient morphism $\tilde{X} \rightarrow Y$ is étale and factors as $\widetilde{X} \rightarrow X \rightarrow Y$. The variety $Y$ has maximal Albanese dimension, is of general type, $P_{1}(Y)=1$, and its Albanese variety is $F_{1} \times F_{2} \times F_{3}$.

Proof. We have a cartesian diagram

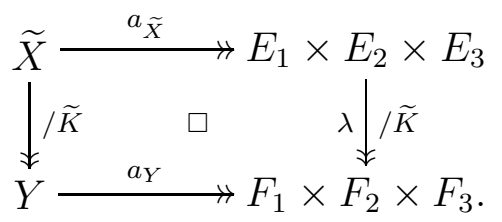

Since the rightmost quotient morphism is étale, so is the leftmost quotient morphism. Since the top morphism is the Albanese mapping of $\widetilde{X}$, the bottom morphism is the Albanese 
mapping of $Y$. Furthermore, since $K$ is a subgroup of $\widetilde{K}$, the leftmost quotient morphism factors as $\widetilde{X} \rightarrow X \rightarrow Y$. Therefore, $P_{1}(Y)=1$.

We now claim that $Y$ is a Chen-Hacon threefold. By Theorem 5.1 (or the diagram (14)), $a_{Y}$ is a $(\mathbf{Z} / 2 \mathbf{Z})^{2}$-Galois covering and by $[\mathrm{P}]$, we can write

$$
a_{Y *} \mathscr{O}_{Y}=\bigoplus_{\chi \in(\mathbf{Z} / 2 \mathbf{Z})^{2 *}} L_{\chi}^{\vee}=\mathscr{O}_{\mathrm{Alb}(Y)} \oplus L_{\chi_{1}}^{\vee} \oplus L_{\chi_{2}}^{\vee} \oplus L_{\chi_{3}}^{\vee},
$$

or equivalently, since $Y$ has rational singularities,

$$
a_{Y *} \omega_{Y}=\mathscr{O}_{F_{1} \times F_{2} \times F_{3}} \oplus L_{\chi_{1}} \oplus L_{\chi_{2}} \oplus L_{\chi_{3}},
$$

where $L_{\chi_{1}}, L_{\chi_{2}}$, and $L_{\chi_{3}}$ are line bundles on $F_{1} \times F_{2} \times F_{3}$. Moreover, by [P], Theorem 2.1, we have the following "building data": there are effective divisors $D_{1}, D_{2}$, and $D_{3}$ on $F_{1} \times F_{2} \times F_{3}$ satisfying:

$$
L_{\chi_{i}}+L_{\chi_{j}} \sim_{\text {lin }} L_{\chi_{k}}+D_{k} \quad \text { and } \quad L_{\chi_{i}}^{2} \sim_{\operatorname{lin}} D_{j}+D_{k}
$$

for any $\{i, j, k\}=\{1,2,3\}$. These data pull back to the analogous building data on $\tilde{X}$, hence $\lambda^{*} D_{i}$ is the pull-back on $E_{1} \times E_{2} \times E_{3}$ of the branch divisor $\Delta_{i} \sim_{\text {lin }} 2 \delta_{i}$ of $\rho_{i}$. It follows that there exists an ample line bundle $\delta_{i}^{\prime}$ on $F_{i}$ which pulls back to $\delta_{i}$ on $E_{i}$ and such that $D_{i}$ is also the pull-back on $F_{1} \times F_{2} \times F_{3}$ of a divisor $\Delta_{i}^{\prime} \sim_{\operatorname{lin}} 2 \delta_{i}^{\prime}$ on $F_{i}$. Let $L_{i}^{\prime}$ be the pull-back on $F_{1} \times F_{2} \times F_{3}$ of $\delta_{i}^{\prime}$. Because of the relations $L_{\chi_{i}}^{2} \sim_{\text {lin }} D_{j}+D_{k}$, we can write

$$
L_{\chi_{i}} \simeq P_{\xi_{i}} \otimes\left(L_{j}^{\prime} \otimes P_{\xi_{i, j}}\right) \otimes\left(L_{k}^{\prime} \otimes P_{\xi_{i, k}}\right)
$$

where $\xi_{i} \in \widehat{E}_{i}, \xi_{i, j} \in \widehat{E}_{j}$, and $\xi_{i, k} \in \widehat{E}_{k}$ are 2-torsion points. From (15) and the fact that $P_{1}(Y)=1$, we deduce $H^{0}\left(E_{i}, L_{\chi_{i}}\right)=0$, hence each $\xi_{i}$ has order 2 and is in the kernel of $\widehat{\lambda}_{i}$. From the relations $L_{\chi_{i}}+L_{\chi_{j}} \sim_{\text {lin }} L_{\chi_{k}}+D_{k}$, we deduce

$$
\xi_{i, k}+\xi_{j, k}=\xi_{k} \quad \text { and } \quad \xi_{i, j}+\xi_{j}=\xi_{k, j} .
$$

Since $\lambda_{1}^{*} \xi_{1}=0$, we may always change $L_{1}^{\prime}$ to $L_{1}^{\prime} \otimes P_{\xi_{1}}$, so we may assume $\xi_{3,1}=0$ and similarly, $\xi_{1,2}=0$ and $\xi_{2,3}=0$. The $\mathscr{O}_{F_{1} \times F_{2} \times F_{3}}$ algebra $a_{Y *} \mathscr{O}_{Y}$ is then the algebra associated to a Chen-Hacon threefold (see (4) ). We conclude that $Y$ is a Chen-Hacon threefold.

\section{A CONJECTURE}

As mentioned in the introduction, we end this article with a conjecture on the possible general structure of smooth projective varieties $X$ of maximal Albanese dimension, of general type, with $\chi\left(X, \omega_{X}\right)=0$.

Conjecture. Let $X$ be a smooth projective variety of maximal Albanese dimension, of general type, with $\chi\left(X, \omega_{X}\right)=0$. Then there exist a smooth projective variety $X^{\prime}$, a morphism $X^{\prime} \rightarrow X$ which is a composition of modifications and abelian étale covers, and a fibration $g: X^{\prime} \rightarrow Y$ with general fiber $F$, such that $0<\operatorname{dim}(Y)<\operatorname{dim}(X)$ and

a) either $g$ is isotrivial;

b) or $\chi\left(F, \omega_{F}\right)=0$. 
Remarks 7.1. 1) Conversely, in the situation b) above, $\chi\left(X, \omega_{X}\right)=0$ ([HP], Proposition 2.5). Moreover, $\operatorname{Alb}(X)$ has at least 4 simple factors by Corollary 3.5.b) and Proposition 4.5.b). Of course, in case a), without further constraints, one might have $\chi\left(X, \omega_{X}\right)>0$, but we were unable to find necessary and sufficient conditions on the isotorivial fibration $g$ (assuming $X$ does not fall into case b)) to ensure $\chi\left(X, \omega_{X}\right)=0$.

3) If we are not in case b), it follows from Lemma 4.6 that if $X^{\prime} \rightarrow X$ is any composition of modifications and abelian étale covers, we have $q\left(X^{\prime}\right)=\operatorname{dim}(X)$ and any morphism from $X^{\prime}$ to a curve of genus $\geq 2$ is constant.

The Ein-Lazarsfeld example (Example 4.1) falls into case a) of the conjecture, and not into case b) by Remark [7.1,1) above. We present an example that falls into case b), but not into case a). It is basically a non-isotrivial fibration whose general fibers are Ein-Lazarsfeld threefolds.

Example 7.2. Consider a smooth projective curve $C$ of genus $\geq 2$, elliptic curves $E_{1}, E_{2}$, and $E_{3}$, and smooth double coverings $S_{j} \rightarrow C \times E_{j}$ ramified along ample divisors. Denote by $\iota_{j}$ the corresponding involution of $S_{j}$. We may moreover assume that the fibrations $f_{j}: S_{j} \rightarrow C$ are all semistable and not isotrivial.

The fourfold $T:=S_{1} \times_{C} S_{2} \times_{C} S_{3}$ has only rational Gorenstein singularities, and so does its quotient $Z$ by the involution $\iota_{1} \times \iota_{2} \times \iota_{3}$. Let $\varepsilon: X \rightarrow Z$ be a desingularization. We have a diagram

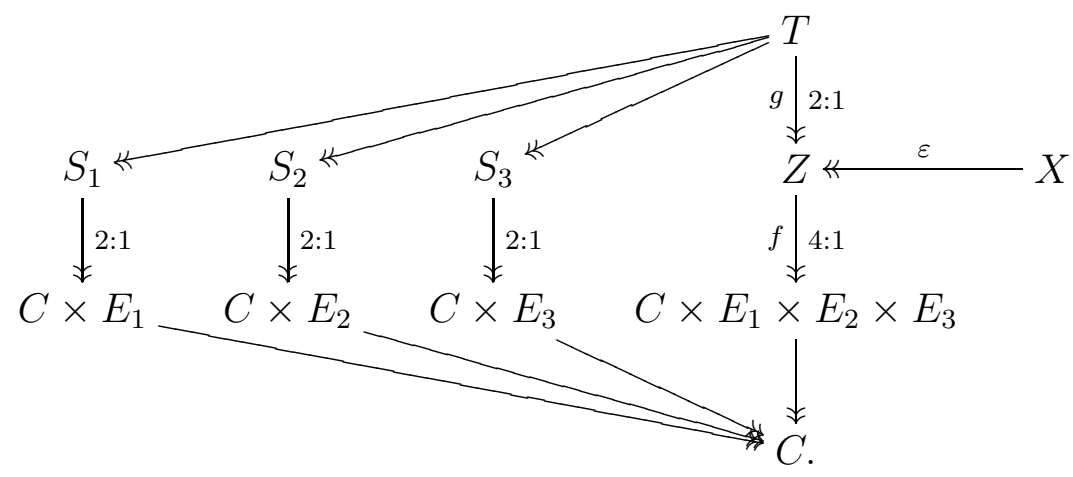

The variety $X$ is of general type and has maximal Albanese dimension because $C \times E_{1} \times$ $E_{2} \times E_{3}$ does. A general fiber of the fibration $X \rightarrow C$ is one of the examples constructed in Example 4.1, hence $\chi\left(X, \omega_{X}\right)=0$ by [HP], Proposition 2.5, and $X$ falls into case b) of the conjecture. One can prove that it does not fall into case a).

\section{REFERENCES}

[AK] Abramovich, D., Karu, K., Weak semistable reduction in characteristic 0, Invent. Math. 139 (2000), 241-273.

[BPV] Barth, W., Peters, C., Van de Ven, A., Compact complex surfaces, Ergebnisse der Mathematik und ihrer Grenzgebiete 4 (1984), Springer Verlag, Berlin-Heidelberg-New York.

[CH1] Chen, J.A., Hacon, C.D., Pluricanonical maps of varieties of maximal Albanese dimension, Math. Ann. 320 (2001), 367-380.

[CH2] Chen, J.A., Hacon, C.D., On the irregularity of the image of the Iitaka fibration, Comm. Algebra 32 (2004), 203-215. 
[EL] Ein, L., Lazarsfeld, R., Singularities of theta divisors and the birational geometry of irregular varieties, J. Amer. Math. Soc. 10 (1997), 243-258.

[GL] Green, M., Lazarsfeld, R., Deformation theory, generic vanishing theorems, and some conjectures of Enriques, Catanese and Beauville, Invent. Math. 90 (1987), 389-407.

[HP] Hacon, C.D., Pardini, R., Birational characterization of products of curves of genus 2, Math. Research Letters 12 (2005), 129-140.

[J] Jiang, Z., Varieties with $q(X)=\operatorname{dim}(X)$ and $P_{2}(X)=2$, eprint arXiv:1010.4709v1, Ann. Scuola Norm. Sup. Pisa, to appear.

[KP] Kebekus, S., Peternell, T., A refinement of Stein factorization and deformations of surjective morphisms, Asian J. Math. 12 (2008), 365-389.

[KKMS] Kempf, G., Knudsen, F., Mumford, D., Saint-Donat, B., Toroidal Embeddings I, Springer Lecture Notes in Mathematics 339, 1973.

[K1] Kollár, J., Shafarevich Maps and Automorphic Forms, Princeton University Press, 1995.

[K2] Kollár, J., Higher direct images of dualizing sheaves. I, Ann. of Math. 123 (1986), 11-42.

[K3] Kollár, J., Subadditivity of the Kodaira dimension: fibers of general type, in Algebraic geometry, Sendai, 1985, 361-398, Adv. Stud. Pure Math. 10, North-Holland, Amsterdam, 1987.

[M] Mori, S., Classification of higher-dimensional varieties, in Algebraic geometry, Bowdoin, 1985 (Brunswick, Maine, 1985), 269-331, Proc. Sympos. Pure Math. 46, Part 1, Amer. Math. Soc., Providence, RI, 1987.

[Mu Mukai, S., Duality between $D(X)$ and $D(\hat{X})$ with its application to Picard sheaves, Nagoya Math. J. 81 (1981), 153-175.

[P] Pardini, R., Abelian covers of algebraic varieties, J. reine angew. Math. 417 (1991), 191-213.

[PP] Pareschi, G., Popa, M., Regularity on abelian varieties I, J. Amer. Math. Soc. 16 (2003), 285-302.

[S] Serrano, F., Isotrivial fibred surfaces, Ann. Mat. Pura Appl. (4) 171 (1996), 63-81.

[Si] Simpson, C., Subspaces of moduli spaces of rank one local systems, Ann. Sci. École Norm. Sup. 26 (1993), 361-401.

[U] Uneo, K., Classification theory of algebraic varieties and compact complex spaces, notes written in collaboration with P. Cherenack, Springer Lecture Notes in Mathematics 439, Berlin-New York, 1975.

[V] Viehweg, E., Positivity of direct image sheaves and applications to famillies of higher dimensional manifolds, ICTP Lecture Notes 6 (2001), 249-284.

Taita Institute for Mathematical Sciences, National Center for Theoretical Sciences, Taipei Office, and Department of Mathematics, 1 Sec. 4, Roosevelt Rd. Taipei 106, TAIWAN

E-mail address: jkchen@math.ntu.edu.tw

Département Mathématiques et Applications, UMR CNRS 8553, École Normale SupéRieure, 45 RUe D'Ulm, 75230 Paris Cedex 05, France

E-mail address: olivier.debarre@ens.fr

Max-Planck-Institut für Mathematik, Vivatsgasse 7, 53111 Bonn, Germany

E-mail address: flipz@mpim-bonn.mpg.de 